\title{
On the boundary motive of a Shimura variety
}

\author{
Jörg Wildeshaus \\ Dedicated to Jozef Steenbrink on the occasion of his 60th birthday
}

\begin{abstract}
Applying the main results of the author's previous work (J. Wildeshaus, The boundary motive: definition and basic properties, Compositio Math. 142 (2006), 631-656), we identify the motives occurring as cones in the co-localization and localization filtrations of the boundary motive of a Shimura variety.
\end{abstract}

\section{Contents}

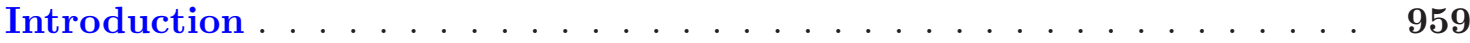

1 Notation for motives . . . . . . . . . . . . . . . . . . . . . 961

2 The completed motive . . . . . . . . . . . . . . . . . . 962

3 Equivariant completed motives . . . . . . . . . . . . . . . . . 964

4 Motives of torus embeddings . . . . . . . . . . . . . . . 969

5 Notation for Shimura varieties . . . . . . . . . . . . . . . . 978

6 Toroidal compactifications of Shimura varieties . . . . . . . . . . . . 979

7 The Baily-Borel compactification of pure Shimura varieties . . . . . 981

References ......................... . . 985

\section{Introduction}

In our previous paper [Wil06], we introduced the boundary motive $\partial M_{\mathrm{gm}}(X)$ of a separated scheme $X$ of finite type over a perfect field. There is an exact triangle

$$
\partial M_{\mathrm{gm}}(X) \longrightarrow M_{\mathrm{gm}}(X) \longrightarrow M_{\mathrm{gm}}^{c}(X) \longrightarrow \partial M_{\mathrm{gm}}(X)[1]
$$

in the category of effective motivic complexes, relating the boundary motive to $M_{\mathrm{gm}}(X)$ and $M_{\mathrm{gm}}^{c}(X)$, the motive of $X$ and its motive with compact support, respectively [Voe00]. This exact triangle should be employed to construct extensions of motives, i.e. classes in motivic cohomology. Almost all of the existing attempts to prove the Beilinson or Bloch-Kato conjectures on special values of $L$-functions necessitate such classes. Furthermore, in these cases, the realizations (Betti, de Rham, étale, etc.) of the classes are constructed inside the cohomology of (non-compact) Shimura varieties, using both:

(1) the respective realizations of our exact triangle

$$
\partial M_{\mathrm{gm}}(X) \longrightarrow M_{\mathrm{gm}}(X) \longrightarrow M_{\mathrm{gm}}^{c}(X) \longrightarrow \partial M_{\mathrm{gm}}(X)[1] ;
$$

(2) an accessible description of the realizations of $\partial M_{\mathrm{gm}}(X)$, when $X$ is a Shimura variety.

Received 4 December 2005, accepted in final form 11 January 2007.

2000 Mathematics Subject Classification 14G35 (primary), 14F42 (secondary).

Keywords: boundary motives, Shimura varieties, toroidal and Baily-Borel compactifications, completed motives.

This journal is (C) Foundation Compositio Mathematica 2007. 


\section{J. Wildeshaus}

This approach is clearly present, for example, in Harder's work on special values. In particular, he gave a conjectural description of the étale realization of $\partial M_{\mathrm{gm}}(X)$, which was eventually proved by Pink [Pin92]. After the work of Harder [Har86], Looijenga-Rapoport and Harris-Zucker [LR91, HZ94], the Hodge theoretic analogue of this description was later established in [BW04].

The aim of this article is to give the motivic version of this description.

Let us develop the statement of our main result. When the base field admits resolution of singularities, then there is a co-localization principle [Wil06, $\S 3$ ]. For any compactification $j: X \hookrightarrow$ $\bar{X}$ of $X$, and any good stratification $\left(i_{Y_{m}}: Y_{m} \hookrightarrow \bar{X}\right)_{m}$ of the boundary $\bar{X}-X$, it relates $\partial M_{\operatorname{gm}}(X)$ to the motives $M_{\mathrm{gm}}\left(Y_{m}, i_{Y_{m}}^{!} j_{!} \mathbb{Z}\right)$ of $Y_{m}$ with coefficients in $i_{Y_{m}}^{!} j_{!} \mathbb{Z}$. When $X$ is smooth, then one also has localization at one's disposal [Wil06, $\S 7$ ], expressing $\partial M_{\mathrm{gm}}(X)$ in terms of the motives $M_{\mathrm{gm}}^{c}\left(Y_{m}, i_{Y_{m}}^{*} j_{*} \mathbb{Z}\right)$ with compact support of $Y_{m}$ and with coefficients in $i_{Y_{m}}^{*} j_{*} \mathbb{Z}$. Both localization and co-localization should be seen as motivic analogues of the well-known principles valid for the classical cohomology theories, relating the cohomology of $\bar{X}-X$ with coefficients in $j_{*} \mathbb{Z}$ to cohomology (with coefficients) of the strata $Y_{m}$.

The main results of this paper identify $M_{\mathrm{gm}}\left(Y_{m}, i_{Y_{m}}^{!} j_{!} \mathbb{Z}\right.$ ) (Theorems 6.1 and 7.1 ) and $M_{\mathrm{gm}}^{c}\left(Y_{m}, i_{Y_{m}}^{*} j_{*} \mathbb{Z}\right)$ (Corollaries 6.2 and 7.2 ) when $X$ is a smooth Shimura variety, and $\bar{X}$ is a toroidal or (when $X$ is pure) the Baily-Borel compactification, equipped with its canonical stratification.

In the toroidal setting, our formulae actually use little from the material contained in the present article. We need two main tools: analytical invariance [Wil06, § 5] relates the toroidal to a toric situation. A local computation then identifies the contribution of a single stratum in a torus embedding (Lemma 4.7 and Corollary 4.9).

For the purpose of this introduction, let us therefore concentrate on the discussion of the BailyBorel compactification $\left(S^{K}\right)^{*}$ of a pure Shimura variety $S^{K}$. Fix a boundary stratum $S_{1}^{K}$. It is (up to an error due to the free action of a finite group) itself a pure Shimura variety. Denote by $i$ the immersion of $S_{1}^{K}$, and by $j$ the open immersion of $S^{K}$ into $\left(S^{K}\right)^{*}$. Invariance under abstract blow-up [Wil06, §4] shows that one can compute $M_{\mathrm{gm}}\left(S_{1}^{K}, i^{!} j ! \mathbb{Z}\right)$ using a toroidal compactification $S^{K}(\mathfrak{S})$ of $S^{K}$. (Invariance under abstract blow-up replaces the usual application of proper base change in classical cohomology theories.) Denote the pre-image of $S_{1}^{K}$ in $S^{K}(\mathfrak{S})$ by $Z^{\prime}$. It is then true that the formal completion of $S^{K}(\mathfrak{S})$ along $Z^{\prime}$ is isomorphic to the quotient by the free action of a certain arithmetic group $\Delta_{1}$ of the formal completion of a relative torus embedding along a union $Z$ of strata. One uses a $\Delta_{1}$-equivariant version of analytical invariance (Theorem 3.14) in order to identify $M_{\mathrm{gm}}\left(S_{1}^{K}, i ! j ! \mathbb{Z}\right)$ with $R \Gamma\left(\Delta_{1}, M_{\mathrm{gm}}(Z, i ! j ! \mathbb{Z})\right)$. Here, $R \Gamma\left(\Delta_{1}, \bullet\right)$ denotes the 'derived' functor of $\Delta_{1}$-invariants on the level of effective motivic complexes; we refer to the first half of $\S 3$ for its definition. The problem is thus reduced to compute the motive with coefficients in $i^{!} j ! \mathbb{Z}$ of a union of strata in a torus embedding. This can be done in some generality (Theorem 4.4). Since the combinatorics of $Z$ is contractible, the result has a particularly easy shape: denoting by $S^{K_{1}}$ the generic stratum of the relative torus embedding, and by $u_{1}$ the relative dimension of $S^{K_{1}}$ over the base, one has

$$
M_{\text {gm }}\left(Z, i^{!} j ! \mathbb{Z}\right) \cong M_{\text {gm }}\left(S^{K_{1}}\right)\left[u_{1}\right]
$$

canonically up to a choice of orientation which will be ignored for the purpose of this introduction. Let us note that $S^{K_{1}}$ is again a (mixed) Shimura variety. This yields the main isomorphism of Theorem 7.1:

$$
M_{\mathrm{gm}}\left(S_{1}^{K}, i^{!} j ! \mathbb{Z}\right) \stackrel{\sim}{\longrightarrow} R \Gamma\left(\Delta_{1}, M_{\mathrm{gm}}\left(S^{K_{1}}\right)\right)\left[u_{1}\right]
$$

The results of [BW04, Pin92] give formulae for the degeneration of mixed (Hodge theoretic or $\ell$-adic) sheaves on a Shimura variety. They induce comparison results on the level of the associated geometric cohomology theories (Betti cohomology in Hodge theory, étale cohomology over the algebraic closure of the reflex field in the $\ell$-adic context). The mixed sheaves are provided by 


\section{On the Boundary MOTIVE OF A SHIMURA VARIETy}

representations of the group underlying the Shimura variety. Theorem 7.1 implies these latter comparison results for the constant representation $\mathbb{Z}$ (see Remark 7.4).

Note that, at present, the $p$-adic version of the descriptions from [BW04, Pin92] is not at one's disposal: it would concern rigid cohomology of closed fibres of a model of the Baily-Borel compactification over a ring of integers. However, it is conceivable to deduce that description from a suitable version of our main result; this would necessitate two ingredients, which at present seem to be within reach: (i) the construction of a version of Voevodsky's motives over Dedekind or at least discrete valuation rings, (ii) the definition of the rigid realization on this category.

To conclude this introduction, let us mention that it is possible to generalize our main results to cover the analogues of certain non-constant representations, namely those occurring as direct images under the projection from relative compactifications of semi-Abelian schemes over the Shimura variety, admitting themselves an interpretation as Shimura varieties (see Remark 7.5). The additional complication caused by this generalization is exclusively notational; the technical tools developed in $\S \S 3$ and 4 cover these cases as well.

\section{Notation for motives}

The notation of the purely motivic sections of this paper $(\S \S 2-4)$ is that of [Wil06] (see in particular [Wil06, §1]), which in turn follows that of [Voe00]. We denote by $k$ the perfect base field over which we work. We write $S c h^{\infty} / k$ for the category of schemes which are separated and locally of finite type over $k, S c h / k$ for the full sub-category of objects which are of finite type over $k$, and $S m / k$ for the full sub-category of objects of $S c h / k$ which are smooth over $k$. Recall the definition of the category $\operatorname{SmCor}(k)$ (see [Voe00, p. 190]): its objects are those of $S m / k$. Morphisms from $Y$ to $X$ are given by the group $c(Y, X)$ of finite correspondences from $Y$ to $X$. The category $S h v_{\text {Nis }}(S m C o r(k))$ of Nisnevich sheaves with transfers [Voe00, Definition 3.1.1] is the category of those contravariant additive functors from $\operatorname{SmCor}(k)$ to Abelian groups, whose restriction to $S m / k$ is a sheaf for the Nisnevich topology. Inside the derived category $D^{-}\left(S h v_{\text {Nis }}(\operatorname{Sm} C o r(k))\right)$ of complexes bounded from above, one defines the full triangulated sub-category $D M_{-}^{\text {eff }}(k)$ of effective motivic complexes over $k$ (see [Voe00, p. 205, Proposition 3.1.13]) as that consisting of objects whose cohomology sheaves are homotopy invariant [Voe00, Definition 3.1.10]. The inclusion of $D M_{-}^{\text {eff }}(k)$ into $D^{-}\left(S h v_{\mathrm{Nis}}(\operatorname{SmCor}(k))\right)$ admits a left adjoint $\mathbf{R} C$, which is induced from the functor

$$
\underline{C}_{*}: S h v_{\mathrm{Nis}}(\operatorname{SmCor}(k)) \longrightarrow C^{-}\left(\operatorname{Sh} v_{\mathrm{Nis}}(\operatorname{Sm} \operatorname{Cor}(k))\right)
$$

which maps $F$ to the simple complex associated to the singular simplicial complex [Voe00, p. 207, Proposition 3.2.3].

One defines a functor $L$ from $S c h^{\infty} / k$ to $S h v_{\text {Nis }}(\operatorname{Sm} \operatorname{Cor}(k))$ : it associates to $X$ the Nisnevich sheaf with transfers $c(\bullet, X)$; note that the above definition of $c(Y, X)$ still makes sense when $X \in S c h^{\infty} / k$ is not necessarily of finite type and smooth. One defines the motive $M_{\mathrm{gm}}(X)$ of $X \in S c h^{\infty} / k$ as $\mathbf{R} C(L(X))$. We shall use the same symbol for $M_{\mathrm{gm}}(X) \in D M_{-}^{\mathrm{eff}}(k)$ and for its canonical representative $\underline{C}_{*}(L(X))$ in $C^{-}\left(S h v_{\mathrm{Nis}}(\operatorname{SmCor}(k))\right)$. There is a second functor $L^{c}$, defined only for $X \in S c h / k$ as the Nisnevich sheaf of quasi-finite correspondences [Voe00, pp. 223-224]. One defines the motive with compact support $M_{\mathrm{gm}}^{c}(X)$ of $X \in S c h / k$ as $\mathbf{R} C\left(L^{c}(X)\right)$.

A second, more geometric approach to motives is that developed in [Voe00, $\S 2.1$, where the triangulated category $D M_{\mathrm{gm}}^{\mathrm{eff}}(k)$ of effective geometrical motives over $k$ is also defined. There is a canonical full triangulated embedding of $D M_{\mathrm{gm}}^{\mathrm{eff}}(k)$ into $D M_{-}^{\mathrm{eff}}(k)$ [Voe00, Theorem 3.2.6], which maps the geometrical motive of $X \in S m / k$ (see [Voe00, Definition 2.1.1]) to $M_{\mathrm{gm}}(X)$. Using this embedding, we consider $M_{\mathrm{gm}}(X)$ as an object of $D M_{\mathrm{gm}}^{\mathrm{eff}}(k)$. Finally, the category $D M_{\mathrm{gm}}(k)$ of geometrical motives over $k$ is obtained from $D M_{\mathrm{gm}}^{\mathrm{eff}}(k)$ by inverting the Tate motive $\mathbb{Z}(1)$ (see [Voe00, p. 192]). 


\section{J. Wildeshaus}

According to [Voe00, Theorem 4.3.1], the functor $D M_{\mathrm{gm}}^{\mathrm{eff}}(k) \rightarrow D M_{\mathrm{gm}}(k)$ is a full triangulated embedding if $k$ admits resolution of singularities.

\section{The completed motive}

The torus embeddings we need to consider later will in general only be locally of finite type. We are thus led to define a new extension of the functor

$$
M_{\mathrm{gm}}: S c h / k \longrightarrow D M_{-}^{\mathrm{eff}}(k)
$$

to a certain sub-category of $S c h^{\infty} / k$. We introduce the notion of completed motive. It will turn out to be very well behaved with respect to free actions of possibly infinite abstract groups, to be considered in the next section. Also, its definition will allow for a straightforward modification of analytical invariance to the geometric context we need to consider later.

Definition 2.1. Let $X \in S c h^{\infty} / k$.

(a) Denote by $\operatorname{Cov}^{(\infty)}(X)$ the system of Zariski coverings $\mathfrak{U}=\left(U_{\alpha}\right)_{\alpha}$ of $X$ such that all $U_{\alpha}$ are in $S c h / k$, and such that for all open subsets $V \subset X$ belonging to $S c h / k$, the set $\left\{\alpha \mid U_{\alpha} \cap V \neq \emptyset\right\}$ is finite.

(b) Denote by $S c h^{(\infty)} / k$ the full sub-category of $S c h^{\infty} / k$ of objects $X$ for which $\operatorname{Cov}^{(\infty)}(X)$ is non-empty.

To the best of the author's knowledge, the class $S c h^{(\infty)} / k$ of schemes has not been considered previously.

Example 2.2. Examples of schemes in $S c h^{\infty} / k$ not belonging to $S c h^{(\infty)} / k$ occur in the context of torus embeddings (see $\S 4$ ). Let $T$ be a split torus over $k$, and $\mathfrak{S}$ a rational partial polyhedral decomposition (see, e.g., [KKMS73, ch. I] or [Pin90, ch. 5]) of $Y_{*}(T)_{\mathbb{R}}$, where $Y_{*}(T)$ denotes the cocharacter group of $T$. It gives rise to an open dense embedding of $T$ into a $k$-scheme $T_{\mathfrak{S}}$ which is locally of finite type. Since $T$ meets any open subset of the torus embedding $T_{\mathfrak{S}}$, this scheme lies in $S c h^{(\infty)} / k$ if and only if $\mathfrak{S}$ is finite.

Note that for $X \in S c h^{(\infty)} / k$, one can find $\mathfrak{U} \in \operatorname{Cov}^{(\infty)}(X)$ such that all members of $\mathfrak{U}$ are affine.

Definition 2.3. Let $Y \hookrightarrow W$ be a closed immersion in $S c h^{\infty} / k$.

(a) Define

$$
L(W / W-Y):=L(W) / L(W-Y)
$$

and

$$
M_{\mathrm{gm}}(W / W-Y):=\mathbf{R} C(L(W / W-Y)) .
$$

(b) Let $\mathfrak{U}_{W}$ be a Zariski covering of an open subset $V$ of $W$, and $X$ a sub-scheme of $W$. Denote by $\mathfrak{U}_{X}$ the induced covering of $V \cap X$.

(c) Denote by $\operatorname{Cov}^{(\infty)}(W)_{Y}$ the system of Zariski coverings $\mathfrak{U}_{W}$ of open subsets of $W$ such that $\mathfrak{U}_{Y}$ is in $\operatorname{Cov}^{(\infty)}(Y)$. (In particular, $\mathfrak{U}_{W}$ covers the whole of $Y$.)

Note that, by definition, $\operatorname{Cov}^{(\infty)}(W)_{Y}$ is non-empty if and only if $Y$ belongs to $S \operatorname{ch} h^{(\infty)} / k$.

Remark 2.4. Let $Y \hookrightarrow W$ be a closed immersion in $S c h^{\infty} / k$, and $\mathfrak{U}_{W}=\left(U_{\alpha}\right)_{\alpha}$ a Zariski covering of an open subset of $W$ containing $Y$. Then $\mathfrak{U}_{W}$ belongs to $\operatorname{Cov}^{(\infty)}(W)_{Y}$ if and only if for all $\beta$, the set $\left\{\alpha \mid U_{\alpha} \cap U_{\beta} \not \subset W-Y\right\}$ is finite. 


\section{ON THE BOUNDARY MOTIVE OF A SHIMURA VARIETY}

For $Y \hookrightarrow W$ as in Definition 2.3 and $\mathfrak{U}_{W}=\left(U_{\alpha}\right)_{\alpha} \in \operatorname{Cov}^{(\infty)}(W)_{Y}$, construct the Čech complex $L \bullet\left(\mathfrak{U}_{W} / \mathfrak{U}_{W-Y}\right)$, concentrated in non-positive degrees, as follows: its $-m$ th term is the direct product of the $L\left(U_{A} / U_{A}-Y\right)$, for all $(m+1)$-fold intersections $U_{A}$ of the $U_{\alpha}$. Note that the differentials are defined thanks to Remark 2.4.

Lemma 2.5. Let $Y \hookrightarrow W$ be a closed immersion in $S c h^{\infty} / k$ and $\mathfrak{U}_{W}=\left(U_{\alpha}\right)_{\alpha \in I} \in \operatorname{Cov}^{(\infty)}(W)_{Y}$.

(a) If $\mathfrak{U}_{Y}$ is finite (and, hence, $Y$ is in $S c h / k$ ), then the augmentation

$$
L^{\bullet}\left(\mathfrak{U}_{W} / \mathfrak{U}_{W-Y}\right) \longrightarrow L(W / W-Y)
$$

is a quasi-isomorphism in $C^{-}\left(\operatorname{Sh} v_{\mathrm{Nis}}(\operatorname{Sm} \operatorname{Cor}(k))\right)$.

(b) Assume that $\mathfrak{V}_{W}=\left(V_{\beta}\right)_{\beta \in J} \in \operatorname{Cov}^{(\infty)}(W)_{Y}$ is a refinement of $\mathfrak{U}_{W}$ :

$$
\forall \beta \in J \exists \alpha \in I, \quad V_{\beta} \subset U_{\alpha} .
$$

Then any refinement map $\iota: J \rightarrow I$ induces a quasi-isomorphism

$$
L^{\bullet}(\iota): L^{\bullet}\left(\mathfrak{V}_{W} / \mathfrak{V}_{W-Y}\right) \longrightarrow L^{\bullet}\left(\mathfrak{U}_{W} / \mathfrak{U}_{W-Y}\right)
$$

in $C^{-}\left(\operatorname{Sh} v_{\mathrm{Nis}}(\operatorname{SmCor}(k))\right)$. Different choices of refinement maps ८ lead to homotopy equivalent morphisms $L^{\bullet}(\iota)$.

Proof. The very last statement is, of course, well known. Note that for $W^{\prime} \in S c h^{\infty} / k$, the sheaf $L\left(W^{\prime}\right)$ is the filtered direct limit of the $L(X)$, with $X$ running through the open sub-schemes of $W^{\prime}$ which are of finite type over $k$. Hence, [Voe00, Proposition 3.1.3] continues to hold for Nisnevich coverings of schemes in $S c h^{\infty} / k$. Part (a) follows from this: observe that since $\mathfrak{U}_{Y}$ is finite, the direct product $\prod_{\alpha \in A} L\left(U_{\alpha} / U_{\alpha}-Y\right)$ equals $L\left(\coprod_{\alpha \in A} U_{\alpha}\right) / L\left(\coprod_{\alpha \in A} U_{\alpha}-Y\right)$, where $A$ denotes the set of indices $\alpha$ for which $U_{\alpha} \not \subset W-Y$. In order to prove part (b), define a third covering

$$
\mathfrak{W}_{W}:=\left(U_{\alpha} \cap V_{\beta}\right)_{\alpha, \beta} .
$$

It is easy to see that it also belongs to $\operatorname{Cov}^{(\infty)}(W)_{Y}$. Furthermore, there are canonical refinement maps from $L \bullet\left(\mathfrak{W}_{W} / \mathfrak{W}_{W-Y}\right)$ to $L \bullet\left(\mathfrak{U}_{W} / \mathfrak{U}_{W-Y}\right)$ and to $L^{\bullet}\left(\mathfrak{V}_{W} / \mathfrak{V}_{W-Y}\right)$. That these are quasi-isomorphisms follows from part (a), applied to each component of $L^{\bullet}\left(\mathfrak{U}_{W} / \mathfrak{U}_{W-Y}\right)$ and $L^{\bullet}\left(\mathfrak{V}_{W} / \mathfrak{V}_{W-Y}\right)$, respectively. Finally, observe that the diagram

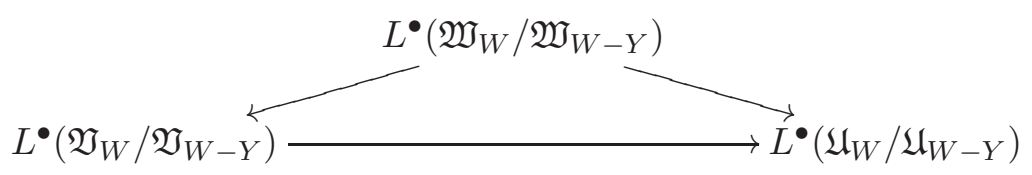

is commutative up to homotopy.

Hence, the following definition makes sense.

Definition 2.6. Let $Y \hookrightarrow W$ be a closed immersion in $S \operatorname{ch}{ }^{\infty} / k$, with $Y \in S \operatorname{ch}(\infty) / k$. The $\operatorname{completed}^{(\infty)}$ motive of $W$ relative to $W-Y$ is defined as

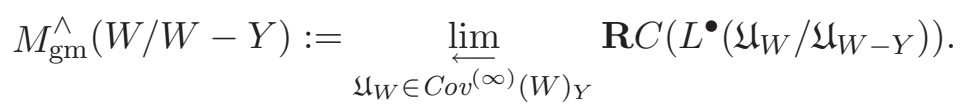

Given that all transition maps in the inverse limit are isomorphisms, our definition should be seen as an attempt to avoid making choices. Of course, $M_{\mathrm{gm}}^{\wedge}(W / W-Y)$ is represented by the simple complex associated to $\underline{C}_{*}\left(L^{\bullet}\left(\mathfrak{U}_{W} / \mathfrak{U}_{W-Y}\right)\right)$, for any $\mathfrak{U}_{W} \in \operatorname{Cov}^{(\infty)}(W)_{Y}$. In particular, we have the following. 


\section{J. Wildeshaus}

Proposition 2.7. Let $Y \hookrightarrow W$ be a closed immersion in $S c h^{\infty} / k$, with $Y \in S c h / k$. Then the augmentation

$$
M_{\mathrm{gm}}^{\wedge}(W / W-Y) \longrightarrow M_{\mathrm{gm}}(W / W-Y)
$$

is an isomorphism in $D M_{-}^{\text {eff }}(k)$.

The case $Y=W$ is important; let us introduce a specific notion.

Definition 2.8. Let $Y \in S c h^{(\infty)} / k$. The completed motive of $Y$ is defined as

$$
M_{\mathrm{gm}}^{\wedge}(Y):=M_{\mathrm{gm}}^{\wedge}(Y / \emptyset) \text {. }
$$

We want to consider the geometric situation in which co-localization works [Wil06, § 3]: fix closed immersions $Y \hookrightarrow Y^{\prime} \hookrightarrow W$ in $S c h^{\infty} / k$. Write $j$ for the open immersion of $W-Y^{\prime}$, and $i_{Y}$ for the closed immersion of $Y$ into $W$. Denote by $\mathfrak{Y}$ the following commutative diagram.

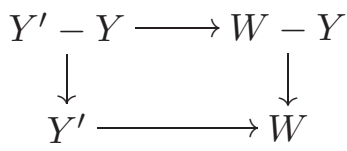

Assume, in addition, that $Y \in S \operatorname{sch}(\infty) / k$. For $\mathfrak{U}_{W} \in \operatorname{Cov}^{(\infty)}(W)_{Y}$, denote by $L^{\bullet}\left(\mathfrak{U}_{\mathfrak{Y}}\right)$ the simple complex associated to

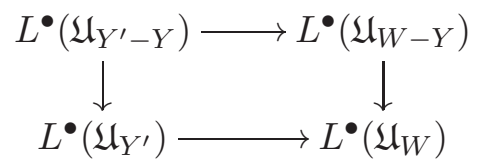

with $L^{\bullet}\left(\mathfrak{U}_{W}\right)$ sitting in degree $(0, *)$. As before, one shows that the following is well defined.

DeFinition 2.9. The completed motive of $Y$ with coefficients in $i_{Y}^{!} j ! \mathbb{Z}$ is defined as

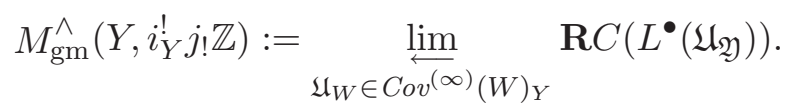

The completed motive $M_{\mathrm{gm}}^{\wedge}\left(Y, i_{Y}^{!} j ! \mathbb{Z}\right)$ is represented by the simple complex associated to $\underline{C}_{*}\left(L \bullet\left(\mathfrak{U}_{\mathfrak{Y}}\right)\right)$, for any $\mathfrak{U}_{W} \in \operatorname{Cov}^{(\infty)}(W)_{Y}$.

Proposition 2.10. In the situation of the definition, there is a canonical exact triangle

$$
M_{\mathrm{gm}}^{\wedge}\left(Y, i_{Y}^{!} j ! \mathbb{Z}\right)[-1] \rightarrow M_{\mathrm{gm}}^{\wedge}\left(Y^{\prime} / Y^{\prime}-Y\right) \rightarrow M_{\mathrm{gm}}^{\wedge}(W / W-Y) \rightarrow M_{\mathrm{gm}}^{\wedge}\left(Y, i_{Y}^{!} j ! \mathbb{Z}\right)
$$

in $D M_{-}^{\mathrm{eff}}(k)$. If $Y \in S c h / k$, then the natural morphism

$$
M_{\mathrm{gm}}^{\wedge}\left(Y, i_{Y}^{!} j ! \mathbb{Z}\right) \longrightarrow M_{\mathrm{gm}}\left(Y, i_{Y}^{!} j ! \mathbb{Z}\right)
$$

is an isomorphism in $D M_{-}^{\mathrm{eff}}(k)$. Here, the right-hand side denotes the motive of $Y$ with coefficients in $i_{Y}^{!} j ! \mathbb{Z}$ (see [Wil06, Definition 3.1]).

Remark 2.11. Definition 2.9 and [Wil06, Definition 3.1] extend to situations where the immersion of $Y$ into $W$ (hence, into $Y^{\prime}$ ) is only locally closed: one replaces $W$ by an open subset containing $Y$ as a closed sub-scheme. Proposition 2.10 continues to hold in this larger generality. We leave the details to the reader.

\section{Equivariant completed motives}

For the application to Shimura varieties, we need to consider the situation of the preceding section under the additional hypothesis of the presence of the action of an abstract group $H$. The result we 


\section{On the Boundary MOTIVE OF A SHIMURA VARIETy}

are aiming at is an $H$-equivariant version of analytical invariance [Wil06, Theorem 5.1]. Let us start by recalling some basic material on the right derived functor of the fixed point functor associated to $H$.

Fix an Abelian category $\mathcal{A}$ and an abstract group $H$. We assume that

(A) $\mathcal{A}$ is closed under arbitrary products, and that

(B) the trivial $\mathbb{Z} H$-module $\mathbb{Z}$ admits a bounded resolution

$$
0 \longrightarrow F_{n} \longrightarrow \cdots \longrightarrow F_{1} \longrightarrow F_{0} \longrightarrow \mathbb{Z} \longrightarrow 0
$$

by free $\mathbb{Z} H$-modules.

Remark 3.1. Recall that $H$ is said to be of type $F L$ if, in addition, the $F_{i}$ in the above resolution can be chosen to be of finite type over $\mathbb{Z} H$.

We shall denote by $H$-A the Abelian category of objects of $\mathcal{A}$ provided with a left $H$-action. If $\gamma \in H$ and $A \in H-\mathcal{A}$, we denote by the same letter $\gamma$ the corresponding automorphism of $A$. Morphisms in $H-\mathcal{A}$ between two objects $A$ and $B$ are those morphisms $f: A \rightarrow B$ in $\mathcal{A}$ commuting with the action of $H$, i.e. such that

$$
f \circ \gamma=\gamma \circ f: A \longrightarrow B, \quad \forall \gamma \in H .
$$

We denote by $e$ the unit element of $H$. By definition, the fixed point functor associated to $H$ is the functor

$$
\Gamma(H, \bullet)=(\bullet)^{H}: H-\mathcal{A} \longrightarrow \mathcal{A}
$$

given by

$$
\Gamma(H, A)=(A)^{H}:=\bigcap_{\gamma \in H} \operatorname{Ker}(e-\gamma)
$$

The condition (A) we put on $\mathcal{A}$ implies that the right derived functor

$$
R \Gamma(H, \bullet): D^{+}(H-\mathcal{A}) \longrightarrow D^{+}(\mathcal{A})
$$

exists (e.g. [BW04, Theorem 3.11, Variant 3.18(a)]). We need to be more precise. Denote by $\mathcal{A} b$ the category of Abelian groups. Recall the definition of the functor Hom : $\mathcal{A} b \times \mathcal{A} \longrightarrow \mathcal{A}$ (see [BW04, $\S 3])$. For $(M, A) \in \mathcal{A} b \times \mathcal{A}$, the object $\operatorname{Hom}(M, A)$ represents the functor associating to $B$ all group morphisms from $M$ to $\operatorname{Hom}_{\mathcal{A}}(B, A)$. If $M$ is free with basis $\left(x_{i}\right)_{i \in I}$, then $\operatorname{Hom}(M, A) \cong \prod_{i \in I} A$. This construction admits an extension to complexes; we denote it by the same symbol Hom : $C^{b}(\mathcal{A} b) \times C^{?}(\mathcal{A}) \longrightarrow C^{?}(\mathcal{A})$, for $? \in\{b,+,-$, blank $\}$. Homotopy equivalent complexes $M_{*}^{1}$ and $M_{*}^{2}$ in $C^{b}(\mathcal{A} b)$ yield homotopy equivalent complexes

$$
\operatorname{Hom}\left(M_{*}^{1}, A^{\bullet}\right) \text { and } \operatorname{Hom}\left(M_{*}^{2}, A^{\bullet}\right)
$$

in $C(\mathcal{A})$, for any $A^{\bullet} \in C(\mathcal{A})$. The same statement is true for the variant Hom : $C^{+}(\mathcal{A} b) \times C^{-}(\mathcal{A}) \longrightarrow$ $C^{-}(\mathcal{A})$ of the functor Hom.

For $\left(M_{*}, A^{\bullet}\right) \in C^{b}(H-\mathcal{A} b) \times C(H-\mathcal{A})$, there is the diagonal action of $H$ on the complex $\operatorname{Hom}\left(M_{*}, A^{\bullet}\right)$ (see [BW04, Definition 3.12]). The sub-complex of (componentwise) invariants is denoted by $\operatorname{Hom}\left(M_{*}, A^{\bullet}\right)^{H}$; note that this complex is in $C^{?}(\mathcal{A} b)$ if $A^{\bullet}$ is in $C^{?}(H-\mathcal{A} b)$. We then have [BW04, Propositon 3.13(b), Variant 3.18(a)] the following.

Proposition 3.2. Let $F_{*} \rightarrow \mathbb{Z}$ be any bounded resolution of the trivial $\mathbb{Z} H$-module $\mathbb{Z}$ by free $\mathbb{Z} H$-modules. Then the functor

$$
R \Gamma(H, \bullet): D^{+}(H-\mathcal{A}) \longrightarrow D^{+}(\mathcal{A})
$$

is induced by $\operatorname{Hom}\left(F_{*}, \bullet\right)^{H}$. 


\section{J. Wildeshaus}

This conclusion would still hold without the boundedness assumption on $F_{*}$. However, we are interested in the following variant, for which the condition (B) we put on $H$ is essential.

Corollary 3.3. Let $F_{*} \rightarrow \mathbb{Z}$ be any bounded resolution of the trivial $\mathbb{Z} H$-module $\mathbb{Z}$ by free $\mathbb{Z} H$-modules. Then the right derived functors

$$
\begin{aligned}
R \Gamma(H, \bullet): D^{b}(H-\mathcal{A}) & \longrightarrow D^{b}(\mathcal{A}), \\
R \Gamma(H, \bullet): D(H-\mathcal{A}) & \longrightarrow D(\mathcal{A})
\end{aligned}
$$

and

$$
R \Gamma(H, \bullet): D^{-}(H-\mathcal{A}) \longrightarrow D^{-}(\mathcal{A})
$$

of $\Gamma(H, \bullet)$ exist and are induced by the functors

$$
\operatorname{Hom}\left(F_{*}, \bullet\right)^{H}: C^{?}(H-\mathcal{A}) \longrightarrow C^{?}(\mathcal{A}) .
$$

In particular, all of the functors denoted $R \Gamma(H, \bullet)$ are compatible under restriction.

Proof. Proposition 3.2 implies that the functor $\Gamma(H, \bullet)$ is of bounded cohomological dimension. Our claim thus follows from [Ver77, Theorem II.2.2.2, Corollary 2].

COROLlary 3.4. We have the following.

(a) Let $? \in\{b,+,-$, blank $\}$. The functor

$$
R \Gamma(H, \bullet): D^{?}(H-\mathcal{A}) \longrightarrow D^{?}(\mathcal{A})
$$

is right adjoint to the functor $\operatorname{Res}_{H}^{1}$ associating to a complex of objects of $\mathcal{A}$ the same complex with trivial $H$-action.

(b) Let $M$ be a free $\mathbb{Z} H$-module, and $A \in H$-A. Then $\operatorname{Hom}(M, A)$ is $\Gamma(H, \bullet)$-acyclic.

Proof. Part (a) results from the fact that the corresponding statement is true for the functor $\Gamma(H, \bullet)$ on $C^{?}(H-\mathcal{A})$, and from the description of $R \Gamma(H, \bullet)$ from Corollary 3.3. For part (b), choose $F_{*} \rightarrow \mathbb{Z}$ as in Corollary 3.3. Observe that, by adjunction,

$$
\operatorname{Hom}\left(F_{*}, \operatorname{Hom}(M, A)\right)^{H}=\operatorname{Hom}\left(F_{*} \otimes_{\mathbb{Z}} M, A\right)^{H} .
$$

The complex $F_{*} \otimes_{\mathbb{Z}} M$ is a free resolution of the free $\mathbb{Z} H$-module $M$. Therefore, the right-hand side is homotopy equivalent to $\operatorname{Hom}(M, A)^{H}$, a complex concentrated in degree zero.

Remark 3.5. Applying the dual of the above method, one shows the existence of the left derived functor

$$
L \Lambda(H, \bullet): D^{-}(H-\mathcal{B}) \longrightarrow D^{-}(\mathcal{B})
$$

of the functor of co-invariants

$$
\Lambda(H, \bullet)=(\bullet)_{H}: H-\mathcal{B} \longrightarrow \mathcal{B}
$$

for any Abelian category $\mathcal{B}$ closed under arbitrary sums. It is left adjoint to the functor $\operatorname{Res}_{H}^{1}$. Note that we do not need to impose condition (B) on the group $H$ as long as we are content to work with complexes bounded from above.

Now we specialize to the case $\mathcal{A}=\operatorname{Sh} v_{\text {Nis }}(\operatorname{Sm} \operatorname{Cor}(k))$. We have

$$
R \Gamma(H, \bullet): D^{-}\left(H-S h v_{\text {Nis }}(\operatorname{SmCor}(k))\right) \longrightarrow D^{-}\left(\operatorname{Sh} v_{\mathrm{Nis}}(\operatorname{SmCor}(k))\right),
$$

and this functor respects the full sub-categories $D^{b}$.

Definition 3.6. We have the following definitions.

(a) An object $F$ of $H$-Sh $v_{\text {Nis }}(S m C o r(k))$ is called homotopy invariant if the underlying object of $\operatorname{Sh} v_{\mathrm{Nis}}(\operatorname{SmCor}(k))$ is homotopy invariant. 


\section{ON THE BOUNDARY MOTIVE OF A SHIMURA VARIETY}

(b) The category $H$-D $M_{-}^{\text {eff }}(k)$ of effective $H$-equivariant motivic complexes over $k$ is the full subcategory of $D^{-}\left(H-S h v_{\text {Nis }}(\operatorname{SmCor}(k))\right)$ consisting of objects whose cohomology sheaves are homotopy invariant.

One shows [Ser01, Corollary 3.5] that one can argue as in the non-equivariant situation [Voe00, $\S 3.1$, to see that $H-D M_{-}^{\text {eff }}(k)$ is a triangulated sub-category of $D^{-}\left(H-S h v_{\text {Nis }}(\operatorname{SmCor}(k))\right)$. It follows from Corollary 3.3 that $R \Gamma(H, \bullet)$ induces

$$
R \Gamma(H, \bullet): H-D M_{-}^{\mathrm{eff}}(k) \longrightarrow D M_{-}^{\mathrm{eff}}(k) .
$$

The functor

$$
\underline{C}_{*}: S h v_{\mathrm{Nis}}(\operatorname{SmCor}(k)) \longrightarrow C^{-}\left(\operatorname{Sh} v_{\mathrm{Nis}}(\operatorname{Sm} \operatorname{Cor}(k))\right)
$$

admits an obvious $H$-equivariant version, and induces

$$
\mathbf{R} C: D^{-}\left(H-S h v_{\mathrm{Nis}}(\operatorname{SmCor}(k))\right) \longrightarrow H-D M_{-}^{\mathrm{eff}}(k) .
$$

One shows [Ser01, Theorem 3.7] that as in the non-equivariant situation, $\mathbf{R} C$ is left adjoint to the inclusion of $H-D M_{-}^{\mathrm{eff}}(k)$ into $D^{-}\left(H-S h v_{\mathrm{Nis}}(\operatorname{Sm} \operatorname{Cor}(k))\right)$. Furthermore, we have the following.

Proposition 3.7. The diagram

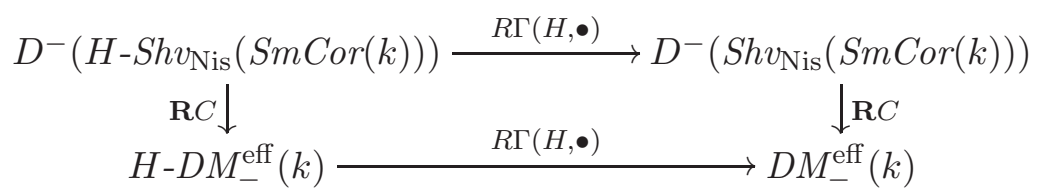

commutes.

Proof. By definition, the functors $\underline{C}_{*}$ and $\operatorname{Hom}\left(F_{*}, \bullet\right)^{H}$ commute.

Remark 3.8. Dually, we get (even without condition (B) on $H$ ) the functor

$$
L \Lambda(H, \bullet): D^{-}\left(H-S h v_{\mathrm{Nis}}(\operatorname{SmCor}(k))\right) \longrightarrow D^{-}\left(\operatorname{Sh} v_{\mathrm{Nis}}(\operatorname{SmCor}(k))\right)
$$

restricting to

$$
L \Lambda(H, \bullet): H-D M_{-}^{\mathrm{eff}}(k) \longrightarrow D M_{-}^{\mathrm{eff}}(k)
$$

and commuting with $\mathbf{R} C$.

Denote by $H$ - $S c h^{\infty} / k$ the category of objects of $S c h^{\infty} / k$ provided with a left $H$-action; similarly for $H$-Sch ${ }^{(\infty)} / k$. Observe that for $X \in H-S c h^{\infty} / k$, the sheaf $L(X)$ is canonically endowed with an $H$-action.

Definition 3.9. Let $X \in H$-Sch ${ }^{\infty} / k$. Define the $H$-equivariant motive $M_{\mathrm{gm}}(X) \in H$-D $M_{-}^{\text {eff }}(k)$ as the image of $L(X) \in H-S h v_{\text {Nis }}(\operatorname{SmCor}(k))$ under $\mathbf{R} C$.

Of course, the natural forgetful functor maps the $H$-equivariant motive to the usual motive.

Remark 3.10. One can (but we do not in this article) consider the full triangulated sub-category $H$-D $M_{\mathrm{gm}}^{\mathrm{eff}}(k)$ of $H$-D $M_{-}^{\mathrm{eff}}(k)$ of effective $H$-equivariant geometrical motives over $k$, i.e. the full triangulated sub-category generated by the $M_{\mathrm{gm}}(X)$, for $X \in H$-Sm $/ k$. The category of $H$-equivariant geometrical motives $H-D M_{\mathrm{gm}}(k)$ is obtained from $H$-D $M_{\mathrm{gm}}^{\mathrm{eff}}(k)$ by inverting $\operatorname{Res}_{H}^{1} \mathbb{Z}(1)$. If $H$ is of type $F L$, then both $R \Gamma(H, \bullet)$ and $L \Lambda(H, \bullet)$ send $H-D M_{\mathrm{gm}}^{\mathrm{eff}}(k)$ to $D M_{\mathrm{gm}}^{\mathrm{eff}}(k)$. Furthermore, by the universal property of $H-D M_{\mathrm{gm}}(k)$, they induce functors

$$
R \Gamma(H, \bullet), L \Lambda(H, \bullet): H-D M_{\mathrm{gm}}(k) \longrightarrow D M_{\mathrm{gm}}(k)
$$




\section{J. Wildeshaus}

right, respectively left adjoint to $\operatorname{Res}_{H}^{1}$. Assume that the category $H-D M_{\mathrm{gm}}(k)$ was known to be a rigid tensor category, in a way compatible with the tensor structure on $D M_{\mathrm{gm}}(k)$ under $\operatorname{Res}_{H}^{1}$. Then duality in $H-D M_{\mathrm{gm}}(k)$ and $D M_{\mathrm{gm}}(k)$ would exchange $R \Gamma(H, \bullet)$ and $L \Lambda(H, \bullet)$ because they are the adjoints of the same tensor functor.

Definition 3.11. Let $X \in H-S c h^{\infty} / k$, and assume that the action of $H$ on $X$ is faithful.

(a) The action of $H$ on $X$ is proper if there is a Zariski covering $\mathfrak{U}$ of $X$ by open affines such that for any $U$ in $\mathfrak{U}$, the set $\{\gamma \in H \mid U \cap \gamma U \neq \emptyset\}$ is finite.

(b) The action of $H$ on $X$ is free if the stabilizer of any point of $X$ is reduced to $\{e\}$.

Note that if $H$ acts properly on $X \in S c h^{\infty} / k$, then the quotient $H \backslash X$ can be formed. Furthermore, if $U_{1}, U_{2} \in S c h / k$ are open sub-schemes of $X$, then the set $\left\{\gamma \in H \mid U_{1} \cap \gamma U_{2} \neq \emptyset\right\}$ is finite. If, in addition, the action of $H$ is free, then there is a Zariski covering $\mathfrak{U}$ of $X$ by open affines such that for any $U$ in $\mathfrak{U}$, we have

$$
\{\gamma \in H \mid U \cap \gamma U \neq \emptyset\}=\{e\} .
$$

Hence, in this case, the projection $\Pi: X \rightarrow H \backslash X$ is a local isomorphism, and $\mathfrak{U}$ induces a Zariski covering $\Pi(\mathfrak{U})$ of $H \backslash X$ by open affines. By definition, a covering $\mathfrak{V}$ of $H \backslash X$ by affines splits $\Pi$ if there is $\mathfrak{U}$ as above such that $\mathfrak{V}=\Pi(\mathfrak{U})$. We leave the proof of the following to the reader.

Proposition 3.12. Let $X \in S c h^{\infty} / k$, with a free and proper action of $H$. Assume that $H \backslash X \in$ $S c h^{(\infty)} / k$ :

(a) there exists a covering $\mathfrak{V} \in \operatorname{Cov}^{(\infty)}(H \backslash X)$ by affines splitting $\Pi$;

(b) $X \in \operatorname{Sch}^{(\infty)} / k$.

We are ready to state the main result of this section. Fix closed immersions $Y_{1} \hookrightarrow W_{1}$ in $H$-Sch $/ k$ and $Y_{2} \hookrightarrow W_{2}$ in $S c h^{\infty} / k$, such that $Y_{2} \in S c h^{(\infty)} / k$, and such that the action of $H$ on $Y_{1}$ is free and proper. Assume we are given an isomorphism $f: Y_{2} \stackrel{\sim}{\longrightarrow} H \backslash Y_{1}$, which extends to an isomorphism

$$
f:\left(W_{2}\right)_{Y_{2}} \stackrel{\sim}{\longrightarrow} H \backslash\left(W_{1}\right)_{Y_{1}},
$$

where $\left(W_{m}\right)_{Y_{m}}$ denotes the formal completion of $W_{m}$ along $Y_{m}, m=1,2$. Note that since the action of $H$ on $Y_{1}$ is free and proper, the same is true for the action on $\left(W_{1}\right)_{Y_{1}}$. In particular, the quotient $\Pi:\left(W_{1}\right)_{Y_{1}} \rightarrow H \backslash\left(W_{1}\right)_{Y_{1}}$ can be formed and is a local isomorphism. Furthermore, Proposition 3.12(b) implies that $Y_{1} \in S c h^{(\infty)} / k$. Using coverings of $Y_{1}$ induced by $f$ and elements of $\operatorname{Cov}^{(\infty)}\left(Y_{2}\right)$, one sees that $M_{\mathrm{gm}}^{\wedge}\left(W_{1} / W_{1}-Y_{1}\right) \in D M_{-}^{\text {eff }}(k)$ has a canonical pre-image under the forgetful functor from $H$ - $D M_{-}^{\mathrm{eff}}(k)$. We use the same symbol $M_{\mathrm{gm}}^{\wedge}\left(W_{1} / W_{1}-Y_{1}\right)$ for this pre-image.

Theorem 3.13. Pull-back of finite correspondences induces an isomorphism

$$
M_{\mathrm{gm}}^{\wedge}\left(W_{2} / W_{2}-Y_{2}\right) \stackrel{\sim}{\longrightarrow} R \Gamma\left(H, M_{\mathrm{gm}}^{\wedge}\left(W_{1} / W_{1}-Y_{1}\right)\right)
$$

in $D M_{-}^{\text {eff }}(k)$, and depending only on $f$.

Proof. Use $f$ to identify $Y_{2}$ and $H \backslash Y_{1}$. Proposition 3.12(a) enables us to choose a covering $\mathfrak{U}_{2} \in$ $\operatorname{Cov}^{(\infty)}\left(Y_{2}\right)$ by affines such that $\Pi: Y_{1} \rightarrow Y_{2}$ is split over $\mathfrak{U}_{2}$. Define $\mathfrak{U}_{1} \in \operatorname{Cov}^{(\infty)}\left(Y_{1}\right)$ as the affine covering induced by $\mathfrak{U}_{2}$ : for any $U_{2} \in \mathfrak{U}_{2}$, its pre-image $\Pi^{-1}\left(U_{2}\right)$ is thus the disjoint union of $|H|$ affines, each of which is isomorphic to $U_{2}$ and belongs to $\mathfrak{U}_{1}$. By definition of the completed motive, $M_{\mathrm{gm}}^{\wedge}\left(W_{m} / W_{m}-Y_{m}\right)$ is represented by

$$
\underline{C}_{*}\left(L \cdot\left(\mathfrak{U}_{W_{m}} / \mathfrak{U}_{W_{m}-Y_{m}}\right)\right),
$$




\section{ON THE BOUNDARY MOTIVE OF A SHIMURA VARIETY}

for any Zariski covering $\mathfrak{U}_{W_{m}}$ of an open subset of $W_{m}$ such that $\mathfrak{U}_{Y_{m}}=\mathfrak{U}_{m}, m=1,2$. Using [Wil06, Theorem 5.5] (including the statements (b) and (c) on compatibility under restriction), one sees that pull-back under $\Pi$ induces canonically

$$
L \cdot\left(\mathfrak{U}_{W_{2}} / \mathfrak{U}_{W_{2}-Y_{2}}\right) \stackrel{\sim}{\longrightarrow} L^{\bullet}\left(\mathfrak{U}_{W_{1}} / \mathfrak{U}_{W_{1}-Y_{1}}\right)^{H} .
$$

Choose for each $U_{2}$ in $\mathfrak{U}_{2}$ a $U_{1}$ in $\mathfrak{U}_{1}$ contained in its pre-image under $\Pi$. This choice induces, again by [Wil06, Theorem 5.5], an isomorphism

$$
\operatorname{Hom}\left(\mathbb{Z} H, L^{i}\left(\mathfrak{U}_{W_{2}} / \mathfrak{U}_{W_{2}-Y_{2}}\right)\right) \stackrel{\sim}{\longrightarrow} L^{i}\left(\mathfrak{U}_{W_{1}} / \mathfrak{U}_{W_{1}-Y_{1}}\right),
$$

for each $i \in \mathbb{Z}$. Here, $L^{i}\left(\mathfrak{U}_{W_{2}} / \mathfrak{U}_{W_{2}-Y_{2}}\right)$ carries the trivial action of $H$. By Corollary 3.4(b), the components of $L \bullet\left(\mathfrak{U}_{W_{1}} / \mathfrak{U}_{W_{1}-Y_{1}}\right)$ are therefore $\Gamma(H, \bullet)$-acyclic. Hence, its $H$-invariants represent $R \Gamma\left(H, L^{\bullet}\left(\mathfrak{U}_{W_{1}} / \mathfrak{U}_{W_{1}-Y_{1}}\right)\right)$. This shows that we even have an isomorphism

$$
L^{\bullet}\left(\mathfrak{U}_{W_{2}} / \mathfrak{U}_{W_{2}-Y_{2}}\right) \stackrel{\sim}{\longrightarrow} R \Gamma\left(H, L \bullet\left(\mathfrak{U}_{W_{1}} / \mathfrak{U}_{W_{1}-Y_{1}}\right)\right)
$$

in $D^{-}\left(\operatorname{Sh} v_{\text {Nis }}(\operatorname{SmCor}(k))\right)$. Our claim then follows from Proposition 3.7.

We add two geometrical data: immersions $Y_{1} \hookrightarrow Y_{1}^{\prime} \hookrightarrow W_{1}$ in $H-S c h{ }^{\infty} / k$ and $Y_{2} \hookrightarrow Y_{2}^{\prime} \hookrightarrow W_{2}$ in $S c h^{\infty} / k$, with $Y_{2} \in S c h^{(\infty)} / k$, such that $Y_{m}^{\prime} \hookrightarrow W_{m}$ are closed, and the action of $H$ on $Y_{1}$ is free and proper. Write $j_{m}$ for the open immersion of $W_{m}-Y_{m}^{\prime}$, and $i_{Y_{m}}$ for the immersion of $Y_{m}$ into $W_{m}$. As above, one notes that there is a canonical pre-image $M_{\mathrm{gm}}^{\wedge}\left(Y_{1}, i_{Y_{1}}^{!} j_{1} ! \mathbb{Z}\right)$ in $H$ - $D M_{-}^{\text {eff }}(k)$ of the completed motive of $Y_{1}$ with coefficients in $i_{Y_{1}}^{!} j_{1} ! \mathbb{Z}$. We have the following $H$-equivariant version of [Wil06, Theorem 5.1].

Theorem 3.14. Assume we are given an isomorphism $f: Y_{2} \stackrel{\sim}{\longrightarrow} H \backslash Y_{1}$, which extends to an isomorphism

$$
f:\left(W_{2}\right)_{Y_{2}} \stackrel{\sim}{\longrightarrow} H \backslash\left(W_{1}\right)_{Y_{1}}
$$

inducing an isomorphism $\left(Y_{2}^{\prime}\right)_{Y_{2}} \cong H \backslash\left(Y_{1}^{\prime}\right)_{Y_{1}}$. Then $f$ induces an isomorphism

$$
M_{\mathrm{gm}}^{\wedge}\left(Y_{2}, i_{Y_{2}}^{!} j_{2 !} \mathbb{Z}\right) \stackrel{\sim}{\longrightarrow} R \Gamma\left(H, M_{\mathrm{gm}}^{\wedge}\left(Y_{1}, i_{Y_{1}}^{!} j_{1 !} \mathbb{Z}\right)\right)
$$

in $D M_{-}^{\text {eff }}(k)$.

Proof. This follows from the fact that the isomorphism $(*)$ in the proof of Theorem 3.13 is compatible with restriction of the $W_{m}$ to $Y_{m}^{\prime}$ (see [Wil06, Theorem 5.5(b)]).

\section{Motives of torus embeddings}

In this section, we consider the following situation: $k$ is a perfect base field, $T$ a split torus over $k, B \in S c h / k$ a reduced scheme, and $X \rightarrow B$ a $T$-torsor. We fix a rational partial polyhedral decomposition (see, e.g., [KKMS73, ch. I] or [Pin90, ch. 5]) $\mathfrak{S}$ of $Y_{*}(T)_{\mathbb{R}}$, where $Y_{*}(T)$ denotes the cocharacter group of $T$, and a non-empty proper subset $\mathfrak{T} \subset \mathfrak{S}$ satisfying the following condition:

$(\alpha)$ whenever $\tau \in \mathfrak{T}$ is a face of $\sigma \in \mathfrak{S}$, then $\sigma$ belongs to $\mathfrak{T}$.

Recall that by definition, any element of $\mathfrak{S}$ is a convex rational polyhedral cone in $Y_{*}(T)_{\mathbb{R}}$, any face of any cone in $\mathfrak{S}$, and the intersection of any two cones in $\mathfrak{S}$ belong to $\mathfrak{S}$, and $\{0\} \in \mathbb{S}$. This implies that $0:=\{0\}$ is a face of any cone in $\mathfrak{S}$, and hence that no such cone contains a non-trivial linear subspace of $Y_{*}(T)_{\mathbb{R}}$. Since $\mathfrak{T}$ is supposed to be a proper subset of $\mathfrak{S}$, the cone 0 does not belong to $\mathfrak{T}$. We assume that:

$(\beta)$ any cone $\sigma$ of $\mathfrak{S}$ is smooth, i.e. the semi-group $\sigma \cap Y_{*}(T)$ can be generated by a subset of a $\mathbb{Z}$-basis of $Y_{*}(T)$. 


\section{J. Wildeshaus}

As described, e.g., in [Pin90, $§ 5.5]$, to the given data are associated: (1) a relative torus embedding $j: X \hookrightarrow X_{\mathfrak{S}}$ identifying $X$ with an open dense sub-scheme of a scheme $X_{\mathfrak{S}}$, which is locally of finite type over $B$; (2) a stratification of $X_{\mathfrak{S}}$ into locally closed reduced sub-schemes $i_{\sigma}: X_{\sigma} \hookrightarrow X_{\mathfrak{S}}$ indexed by the cones $\sigma \in \mathfrak{S}$, and of finite type over $B$. This stratification allows us to associate to any locally closed subset $\mathfrak{V}$ of $\mathfrak{S}$ the locally closed reduced sub-scheme $X_{\mathfrak{V}}$ of $X_{\mathfrak{S}}$ which settheoretically is the union of the $X_{\sigma}$, with $\sigma \in \mathfrak{V}$; here, a subset $\mathfrak{V}$ of $\mathfrak{S}$ is called locally closed if the relations $\left(\nu \in \mathfrak{V}\right.$ is a face of $\sigma \in \mathfrak{S}$ ) and ( $\sigma$ is a face of $\mu \in \mathfrak{V}$ ) imply that $\sigma \in \mathfrak{V}$. The scheme $X_{\mathfrak{V}}$ is of finite type over $B$ if and only if $\mathfrak{V}$ is finite. In particular, the above data define: (3) a sub-scheme $i_{\mathfrak{T}}: X_{\mathfrak{T}} \hookrightarrow X_{\mathfrak{S}}$ contained in the complement of $X=X_{0}$. Note that $X_{\mathfrak{T}}$ is closed in $X_{\mathfrak{S}}$ because of condition $(\alpha)$, and that $X_{\mathfrak{S}}$ is smooth over $B$ because of condition $(\beta)$.

We add one more condition on the given data. In order to formulate it, define

$$
D:=\bigcup_{\tau \in \mathfrak{T}} \tau^{\circ} \subset Y_{*}(T)_{\mathbb{R}}
$$

where for each cone $\tau$ we denote by $\tau^{\circ}$ the topological interior of $\tau$ inside the linear subspace of $Y_{*}(T)_{\mathbb{R}}$ generated by $\tau$. The subset $D$ of $Y_{*}(T)_{\mathbb{R}}$ is endowed with the induced topology. Note that $0 \notin D$ since $0 \notin \mathfrak{T}$, and that $D$ is conic in the sense that multiplication by strictly positive real numbers on $Y_{*}(T)_{\mathbb{R}}$ preserves $D$. We assume that:

$(\gamma)$ every point of $D$ admits a neighbourhood $U$ such that $U \cap \tau \neq \emptyset$ for only a finite number of $\tau \in \mathfrak{T}$.

Note that $(\gamma)$ implies that every $\tau \in \mathfrak{T}$ is a face of only finitely many $\sigma \in \mathfrak{T}$. Hence, the closure of any $X_{\tau}$, for $\tau \in \mathfrak{T}$, is of finite type over $B$. Furthermore, writing

$$
\overline{\{\sigma\}}:=\{\nu \in \mathfrak{S} \mid \nu \text { face of } \sigma\}
$$

for any cone $\sigma$ of $\mathfrak{S}$, we have the following.

Proposition 4.1. We have:

(a) the scheme $X_{\mathfrak{T}}$ lies in $\operatorname{Sch}^{(\infty)} / k$;

(b) the system $\left(X_{\overline{\{\tau\}}}\right)_{\tau \in \mathfrak{T}}$ belongs to $\operatorname{Cov}^{(\infty)}\left(X_{\mathfrak{S}}\right)_{X_{\mathfrak{T}}}$.

This result should be compared with Example 2.2.

Proof of Proposition 4.1. The sub-scheme $X_{\overline{\{\tau\}}} \subset X_{\mathfrak{S}}$ is open since $\overline{\{\tau\}}$ contains $\nu$ whenever $\nu$ is a face of a cone in $\overline{\{\tau\}}$. Furthermore, $X_{\overline{\{\tau\}}}$ is of finite type over $B$ since $\overline{\{\tau\}}$ is finite. Finally, the condition from Remark 2.4 is satisfied because any element of $\overline{\{\tau\}}$ is a face of only finitely many cones.

Thus, we can consider $M_{\mathrm{gm}}^{\wedge}\left(X_{\mathfrak{T}}, i_{\mathfrak{T}}^{!} j ! \mathbb{Z}\right) \in D M_{-}^{\text {eff }}(k)$, the completed motive of $X_{\mathfrak{T}}$ with coefficients in $i_{\mathfrak{T}} j ! \mathbb{Z}$ defined in Definition 2.9. The aim of this section is to identify this object.

Note that the open covering $\left(X_{\overline{\{\tau\}} \cap \mathfrak{T}}\right)_{\tau \in \mathfrak{T}}$ of $X_{\mathfrak{T}}$ corresponds to a closed covering $(\tau \cap D)_{\tau \in \mathfrak{T}}$ of $D$ with the same combinatorics. Denote by $C_{*}\left((\tau \cap D)_{\tau \in \mathfrak{T}}, \mathbb{Z}\right)$ the homological Cech complex associated to this covering. Its $p$ th term is the direct sum of the $H_{0}\left(\tau_{0} \cap \cdots \cap \tau_{p} \cap D, \mathbb{Z}\right)$, for all $(p+1)$-fold intersections $\tau_{0} \cap \cdots \cap \tau_{p}$ of the $\tau_{i}$, with $\tau_{i} \in \mathfrak{T}$. Note that $\tau_{0} \cap \cdots \cap \tau_{p} \cap D$ is either contractible or empty according to whether $\tau_{0} \cap \cdots \cap \tau_{p}$ is a cone in $\mathfrak{T}$ or not. In particular, the above Čech complex computes singular homology of $D$ with coefficients in $\mathbb{Z}$. We define $C_{*}(\mathfrak{T}, \mathbb{Z})=\left(C_{p}, \delta_{p}\right)$, the co-cellular complex of $D$ with respect to the stratification induced by $\mathfrak{T}$, as follows: we set

$$
C_{p}:=\bigoplus_{\tau \in \mathfrak{T}_{-p}} \mathbb{Z}(\tau)
$$




\section{ON THE BOUNDARY MOTIVE OF A SHIMURA VARIETY}

where $\mathfrak{T}_{q}:=\{\tau \in \mathfrak{T} \mid \operatorname{dim} \tau=q\}$, and $\mathbb{Z}(\tau)$ is the group of orientations of $\tau$, i.e. the free Abelian group of rank one defined as the maximal exterior power

$$
\operatorname{det}(\mathbb{Z}[\nu \in \mathfrak{S} \mid \operatorname{dim} \nu=1, \nu \text { face of } \tau]) .
$$

Any ordering of the set $\{\nu \in \mathfrak{S} \mid \operatorname{dim} \nu=1, \nu$ face of $\tau\}$ gives a generator of $\mathbb{Z}(\tau)$. A change of the ordering by a permutation multiplies the generator by the signature of the permutation. The tensor square $\mathbb{Z}(\tau)^{\otimes 2}$ is canonically isomorphic to $\mathbb{Z}$. The restriction of the differential $\delta_{p}: C_{p+1} \rightarrow C_{p}$ to $\mathbb{Z}(\tau)$, for $\tau \in \mathfrak{T}_{-(p+1)}$ is defined as follows: fix an ordering $\nu_{1} \prec \cdots \prec \nu_{-(p+1)}$ of the faces of dimension one of $\tau$. For $\eta \in \mathfrak{T}_{-p}$, the component of $\delta_{p}$ involving $\eta$ is zero if $\tau$ is not a face of $\eta$. If $\tau$ is a face of $\eta$, then $\eta$ has exactly one more face $\nu_{-p}$ of dimension one. Define $\mathbb{Z}(\tau) \longrightarrow \mathbb{Z}(\eta)$ by $z \otimes\left(\nu_{1} \prec \cdots \prec \nu_{-(p+1)}\right) \mapsto z \otimes\left(\nu_{1} \prec \cdots \prec \nu_{-(p+1)} \prec \nu_{-p}\right)$. This definition is obviously independent of the choice of the ordering of the set $\{\nu \in \mathfrak{S} \mid \operatorname{dim} \nu=1, \nu$ face of $\tau\}$. Observe that for any cone $\eta$ in $\mathfrak{T}$ of dimension $r$ having a face $\tau$ of dimension $r-2$ which still belongs to $\mathfrak{T}$, there are exactly two faces of $\eta$ of dimension $r-1$ having $\tau$ as face, and belonging to $\mathfrak{T}$. This shows that $C_{*}(\mathfrak{T}, \mathbb{Z})$ is indeed a complex.

Remark 4.2. One sees that $C_{*}\left(\mathfrak{T}^{\prime}, \mathbb{Z}\right)$ can be defined for any locally closed subset $\mathfrak{T}^{\prime}$ of $\mathfrak{T}$. It is contravariantly functorial with respect to inclusions $\iota: \mathfrak{T}^{\prime \prime} \hookrightarrow \mathfrak{T}^{\prime}$ of two such subsets: for $\tau \in \mathfrak{T}^{\prime}$, the map $\iota^{*}$ is the identity on $\mathbb{Z}(\tau)$ if $\tau \in \mathfrak{T}^{\prime \prime}$, and is zero if $\tau \notin \mathfrak{T}^{\prime \prime}$.

Define $D_{\mathbb{R}}$ to be the sub-vector space of $Y_{*}(T)_{\mathbb{R}}$ generated by $D$, and let $\mathbb{Z}\left(D_{\mathbb{R}}\right)$ denote the group of orientations of $D_{\mathbb{R}}$. Set $d:=\operatorname{dim}_{\mathbb{R}} D_{\mathbb{R}}$.

Proposition 4.3. Assume that $D$ is open in $D_{\mathbb{R}}$.

(a) The co-cellular complex of $D$ computes singular homology of $D$. More precisely, there is a canonical isomorphism

$$
H_{p}\left(C_{*}(\mathfrak{T}, \mathbb{Z})\right) \stackrel{\sim}{\longrightarrow} H_{p+d}(D, \mathbb{Z}) \otimes_{\mathbb{Z}} \mathbb{Z}\left(D_{\mathbb{R}}\right)
$$

for all $p \in \mathbb{Z}$.

(b) If $D$ is contractible, then $C_{*}(\mathfrak{T}, \mathbb{Z})$ is a (bounded) resolution of $\mathbb{Z}\left(D_{\mathbb{R}}\right)[-d]$.

Proof. We leave it to the reader to canonically represent $C_{*}(\mathfrak{T}, \mathbb{Z})$ as a sub-quotient of the shift by -d of $C_{*}\left((\tau \cap D)_{\tau \in \mathfrak{T}}, \mathbb{Z}\right) \otimes_{\mathbb{Z}} \mathbb{Z}\left(D_{\mathbb{R}}\right)$; note that any choice of orientation of $D_{\mathbb{R}}$ induces orientations on all maximal cones of $\mathfrak{T}$. The hypothesis on $D$ implies that any cone in $\mathfrak{T}$ of dimension $d-p$, for $p \geqslant 0$, is the intersection of precisely $p+1$ maximal cones in $\mathfrak{T}$. This means that components $H_{0}\left(\tau_{0} \cap \cdots \cap \tau_{p} \cap D, \mathbb{Z}\right)$ of degree $p$ in $C_{*}\left((\tau \cap D)_{\tau \in \mathfrak{T}}, \mathbb{Z}\right)$, but with

$$
\operatorname{dim}\left(\tau_{0} \cap \cdots \cap \tau_{p}\right)<d-p
$$

occur also in degree $p+1$. Using this, one shows that $C_{*}(\mathfrak{T}, \mathbb{Z})$ is quasi-isomorphic to the Cech complex, tensored with $\mathbb{Z}\left(D_{\mathbb{R}}\right)$ and shifted by $-d$.

Recall the definition of the functor

$$
\text { Hom : } C^{b}(\mathcal{A} b) \times S h v_{\text {Nis }}(\operatorname{SmCor}(k)) \longrightarrow C^{b}\left(S h v_{\text {Nis }}(\operatorname{SmCor}(k))\right)
$$

from $\S 3$. We now state the main result of this section.

Theorem 4.4. We have the following.

(a) There is a canonical isomorphism

$$
M_{\mathrm{gm}}^{\wedge}\left(X_{\mathfrak{T}}, i_{\mathfrak{T}}^{!} j ! \mathbb{Z}\right) \stackrel{\sim}{\longrightarrow} \mathbf{R} C\left(\operatorname{Hom}\left(C_{*}(\mathfrak{T}, \mathbb{Z}), L(X)\right)\right)
$$

in $D M_{-}^{\mathrm{eff}}(k)$. 


\section{J. Wildeshaus}

(b) If $\mathfrak{T}$ is finite, then

$$
M_{\mathrm{gm}}\left(X_{\mathfrak{T}}, i_{\mathfrak{T}} j ! \mathbb{Z}\right) \stackrel{\sim}{\longrightarrow} \mathbf{R} C\left(\operatorname{Hom}\left(C_{*}(\mathfrak{T}, \mathbb{Z}), L(X)\right)\right)
$$

in $D M_{-}^{\mathrm{eff}}(k)$.

(c) If $D$ is contractible and open in $D_{\mathbb{R}}$, then

$$
M_{\mathrm{gm}}^{\wedge}\left(X_{\mathfrak{T}}, i_{\mathfrak{T}}^{!} j ! \mathbb{Z}\right) \stackrel{\sim}{\longrightarrow} \operatorname{Hom}\left(\mathbb{Z}\left(D_{\mathbb{R}}\right), M_{\mathrm{gm}}(X)\right)[d]
$$

in $D M_{-}^{\mathrm{eff}}(k)$. In particular, any choice of an orientation of $D_{\mathbb{R}}$ induces an isomorphism

$$
M_{\mathrm{gm}}^{\wedge}\left(X_{\mathfrak{T}}, i_{\mathfrak{T}}^{!} j ! \mathbb{Z}\right) \cong M_{\mathrm{gm}}(X)[d] .
$$

Of course, parts (b) and (c) follow from part (a), together with Propositions 2.10 and 4.3(b). Actually, the proof will show that the above isomorphisms satisfy strong functoriality properties in the geometric data we fixed in the beginning. For our application to the Baily-Borel compactification of pure Shimura varieties $(\S 7)$, we need to spell out functoriality in a particular situation: in addition to the set of data $(1)-(3)$ and conditions $(\alpha)-(\gamma)$ fixed so far, we assume that we are given (4) an abstract group $H$ satisfying condition (B) from $\S 3$, acting on $B, X$, and $T$ in a way compatible with the group and torsor structures, stabilizing $\mathfrak{S}$ and $\mathfrak{T}$, and satisfying the following condition.

$(\delta)$ The action of $H$ on the set $\mathfrak{T}$ is free.

This implies that the induced action of $H$ on $X_{\mathfrak{T}}$ is free and proper. As was explained before Theorem 3.14, this allows us to define $M_{\mathrm{gm}}^{\wedge}\left(X_{\mathfrak{T}}, i_{\mathfrak{T}}^{!} j ! \mathbb{Z}\right) \in H$-D $M_{-}^{\text {eff }}(k)$. Observe that the right-hand sides of the isomorphisms from Theorem 4.4 also define objects in $H$ - $D M_{-}^{\text {eff }}(k)$; note that $H$ acts on $\mathbb{Z}\left(D_{\mathbb{R}}\right)$ via a character of order at most two. Our proof of Theorem 4.4 will show the following.

Complement 4.5. We have the following.

(a) There is a canonical isomorphism

$$
M_{\mathrm{gm}}^{\wedge}\left(X_{\mathfrak{T}}, i_{\mathfrak{T}} j ! \mathbb{Z}\right) \stackrel{\sim}{\longrightarrow} \mathbf{R} C\left(\operatorname{Hom}\left(C_{*}(\mathfrak{T}, \mathbb{Z}), L(X)\right)\right)
$$

in $H-D M_{-}^{\text {eff }}(k)$, which is mapped to that from Theorem 4.4(a) under the forgetful functor.

(b) If $D$ is contractible and open in $D_{\mathbb{R}}$, then

$$
M_{\mathrm{gm}}^{\wedge}\left(X_{\mathfrak{T}}, i_{\mathfrak{T}} j ! \mathbb{Z}\right) \stackrel{\sim}{\longrightarrow} \operatorname{Hom}\left(\mathbb{Z}\left(D_{\mathbb{R}}\right), M_{\mathrm{gm}}(X)\right)[d]
$$

in $H-D M_{-}^{\mathrm{eff}}(k)$. This isomorphism is compatible with the isomorphism from Theorem 4.4(c).

Note that under the above conditions, the action of $H$ is also free and proper on the formal completions $\left(X_{\mathfrak{S}-\{0\}}\right)_{X_{\mathfrak{T}}}$ and $\left(X_{\mathfrak{S}}\right)_{X_{\mathfrak{T}}}$ of $X_{\mathfrak{S}-\{0\}}$ and $X_{\mathfrak{S}}$ along $X_{\mathfrak{T}}$. Hence, the geometric quotients $H \backslash X_{\mathfrak{T}}, H \backslash\left(X_{\mathfrak{S}-\{0\}}\right)_{X_{\mathfrak{T}}}$, and $H \backslash\left(X_{\mathfrak{S}}\right)_{X_{\mathfrak{T}}}$ exist, and $X_{\mathfrak{T}} \rightarrow H \backslash X_{\mathfrak{T}},\left(X_{\mathfrak{S}-\{0\}}\right)_{X_{\mathfrak{T}}} \rightarrow H \backslash\left(X_{\mathfrak{S}-\{0\}}\right)_{X_{\mathfrak{T}}}$, and $\left(X_{\mathfrak{S}}\right)_{X_{\mathfrak{T}}} \rightarrow H \backslash\left(X_{\mathfrak{S}}\right)_{X_{\mathfrak{T}}}$ are local isomorphisms.

We make one last extension of the data: we assume that we are given (5) separated reduced schemes $S, S_{\mathfrak{S}}$, and $S_{\mathfrak{T}}$, which are locally of finite type over $k$, and related by an open immersion $S \hookrightarrow S_{\mathfrak{S}}$ and a closed immersion $S_{\mathfrak{T}} \hookrightarrow S_{\mathfrak{S}}$ identifying $S_{\mathfrak{T}}$ with a closed sub-scheme of the reduced scheme $S_{\mathfrak{S}-\{0\}}:=S_{\mathfrak{S}}-S$. We assume that $S_{\mathfrak{T}} \in S c h^{(\infty)} / k$. By abuse of notation, we again use the symbols $j$ and $i_{\mathfrak{T}}$ for these immersions. We fix (6) a triple of compatible isomorphisms

$$
H \backslash\left(X_{\mathfrak{S}}\right)_{X_{\mathfrak{T}}} \stackrel{\sim}{\longrightarrow}\left(S_{\mathfrak{S}}\right)_{S_{\mathfrak{T}}}, \quad H \backslash\left(X_{\mathfrak{S}-\{0\}}\right)_{X_{\mathfrak{T}}} \stackrel{\sim}{\longrightarrow}\left(S_{\mathfrak{S}-\{0\}}\right)_{S_{\mathfrak{T}}}, \quad H \backslash X_{\mathfrak{T}} \stackrel{\sim}{\longrightarrow} S_{\mathfrak{T}},
$$

all denoted by the same symbol $f$, where $(\bullet)_{S_{\mathfrak{T}}}$ denotes the formal completions along $S_{\mathfrak{T}}$. Complement 4.5 and Theorem 3.14 then imply the following. 
THEOREM 4.6. We have the following.

(a) Under the above hypotheses, $f$ induces an isomorphism

$$
M_{\mathrm{gm}}^{\wedge}\left(S_{\mathfrak{T}}, i_{\mathfrak{T}}^{!} j ! \mathbb{Z}\right) \stackrel{\sim}{\rightarrow} \mathbf{R} C\left(\operatorname{Hom}\left(C_{*}(\mathfrak{T}, \mathbb{Z}), L(X)\right)^{H}\right)
$$

in $D M_{-}^{\mathrm{eff}}(k)$.

(b) If $H \backslash \mathfrak{T}$ is finite (hence $S_{\mathfrak{T}} \in S c h / k$ ), then

$$
M_{\mathrm{gm}}\left(S_{\mathfrak{T}}, i_{\mathfrak{T}} j ! \mathbb{Z}\right) \stackrel{\sim}{\longrightarrow} \mathbf{R} C\left(\operatorname{Hom}\left(C_{*}(\mathfrak{T}, \mathbb{Z}), L(X)\right)^{H}\right)
$$

in $D M_{-}^{\text {eff }}(k)$.

(c) If $D$ is contractible and open in $D_{\mathbb{R}}$, then

$$
M_{\mathrm{gm}}^{\wedge}\left(S_{\mathfrak{T}}, i_{\mathfrak{T}}^{!} j ! \mathbb{Z}\right) \stackrel{\sim}{\longrightarrow} R \Gamma\left(H, \operatorname{Hom}\left(\mathbb{Z}\left(D_{\mathbb{R}}\right), M_{\mathrm{gm}}(X)\right)\right)[d]
$$

in $D M_{-}^{\text {eff }}(k)$.

Proof. The isomorphisms in $D M_{-}^{\text {eff }}(k)$ are those of Theorem 3.14. That the complexes in (a), (b) represent the right classes in $D M_{-}^{\text {eff }}(k)$ results from Corollary 3.4(b) and hypothesis $(\delta)$ on the action of $H$ on $\mathfrak{T}$.

Note that hypotheses (b) and (c) of Theorem 4.6 together imply that $H$ is of type $F L$.

We prepare the proof of Theorem 4.4 (and forget the data (4)-(6) and condition $(\delta)$ ). We need to identify the contribution of each $X_{\overline{\{\sigma\}}}$ to the completed motive $M_{\mathrm{gm}}^{\wedge}\left(X_{\mathfrak{T}}, i_{\mathfrak{T}}^{!} j_{!} \mathbb{Z}\right)$. Write $n:=\operatorname{dim} T$ and fix a cone $\sigma \in \mathfrak{S}$ of dimension $r$. The finite subset $\overline{\{\sigma\}}$ of $\mathfrak{S}$ corresponds to an open sub-scheme $X_{\overline{\{\sigma\}}} \subset X_{\mathfrak{S}}$ which inherits the stratification, and which is of finite type over $B$. For any face $\nu$ of $\sigma$, the scheme $X_{\overline{\{\nu\}}}$ is open in $X_{\overline{\{\sigma\}}}$, and contains $X_{\nu}$ as unique closed stratum. Use the symbol $j$ to denote also the open immersion of $X$ into $X_{\overline{\{\sigma\}}}$. Zariski-locally over $B, j: X \hookrightarrow X_{\overline{\{\sigma\}}}$ is isomorphic to $\mathbb{G}_{m}^{n} \hookrightarrow \mathbb{G}_{m}^{n-r} \times \mathbb{A}^{r}$, and the stratification is the natural stratification of $\mathbb{A}^{n}$ according to the vanishing (respectively, non-vanishing) of coordinates. Let us start with the identification of $M_{\mathrm{gm}}\left(X_{\sigma}, i_{\sigma}^{!} j ! \mathbb{Z}\right)$. By definition, this is the motive $M_{\mathrm{gm}}\left(\mathfrak{Y}_{\sigma}\right)$ associated to the diagram

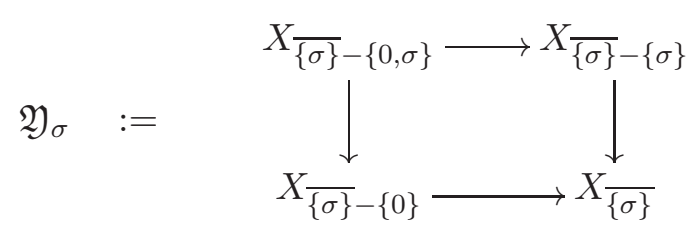

as in [Wil06, Convention 1.2]. The component $X_{\overline{\{\sigma\}}}$ sits in total degree zero. Our main computational tool is the following.

Lemma 4.7. Let $\nu \in \overline{\{\sigma\}}$. The immersion $X_{\overline{\{\nu\}}-\{0\}} \hookrightarrow X_{\overline{\{\nu\}}}$ induces an isomorphism

$$
M_{\mathrm{gm}}\left(X_{\overline{\{\nu\}}-\{0\}}\right) \stackrel{\sim}{\longrightarrow} M_{\mathrm{gm}}\left(X_{\overline{\{\nu\}}}\right)
$$

in $D M_{-}^{\mathrm{eff}}(k)$ provided that $\nu \neq 0$.

Proof. The Mayer-Vietoris property [Voe00, Proposition 3.1.3] for the functor $L$ shows that we may assume that $X=T \times_{k} B$. For a suitable choice of identification $\mathbb{G}_{m}^{n} \cong T$, the immersion $X_{0} \hookrightarrow X_{\overline{\{\nu\}}}$ is isomorphic to $\mathbb{G}_{m}^{n} \longleftrightarrow \mathbb{G}_{m}^{n-i} \times \mathbb{A}^{i}$, with $i \neq 0$. We may reduce to the case $i=n$. Now observe that $\mathbb{A}^{n}-\mathbb{G}_{m}^{n} \longleftrightarrow \mathbb{A}^{n}$ is a homotopy equivalence for $n \neq 0$ (in fact, both sides are homotopic to a point).

In order to compute the motive $M_{\mathrm{gm}}\left(\mathfrak{Y}_{\sigma}\right)$, consider the upper line $X_{\overline{\{\sigma\}}-\{0, \sigma\}} \longrightarrow X_{\overline{\{\sigma\}}-\{\sigma\}}$ of $\mathfrak{Y}_{\sigma}$, and the finite covering $X_{\overline{\{\sigma\}}-\{\sigma\}}=\bigcup_{\nu \in \overline{\{\sigma\}}^{1}} X_{\overline{\{\nu\}}}$, where $\overline{\{\sigma\}}^{1} \subset \overline{\{\sigma\}}$ denotes the subset 


\section{J. Wildeshaus}

(of cardinality $r$ ) of faces $\nu$ of codimension one in $\sigma$. For $\nu_{1}, \ldots, \nu_{p} \in \overline{\{\sigma\}}^{1}$, we have

$$
\bigcap_{i=1}^{p} X_{\overline{\left\{\nu_{i}\right\}}}=X_{\overline{\{\nu\}}}, \quad \text { with } \nu=\bigcap_{i} \nu_{i} .
$$

Fix an ordering of $\{\nu \in \mathfrak{S} \mid \operatorname{dim} \nu=1, \nu$ face of $\sigma\}$. This induces an ordering of $\overline{\{\sigma\}}^{1}$. Define the double complex $L\left(\mathfrak{Y}_{\sigma}^{\prime}\right)$ of Nisnevich sheaves as follows: first consider the Cech complex associated to the above covering, and to the induced covering of $X_{\overline{\{\sigma\}}-\{0, \sigma\}}$, then add the lower line of $L\left(\mathfrak{Y}_{\sigma}\right)$.

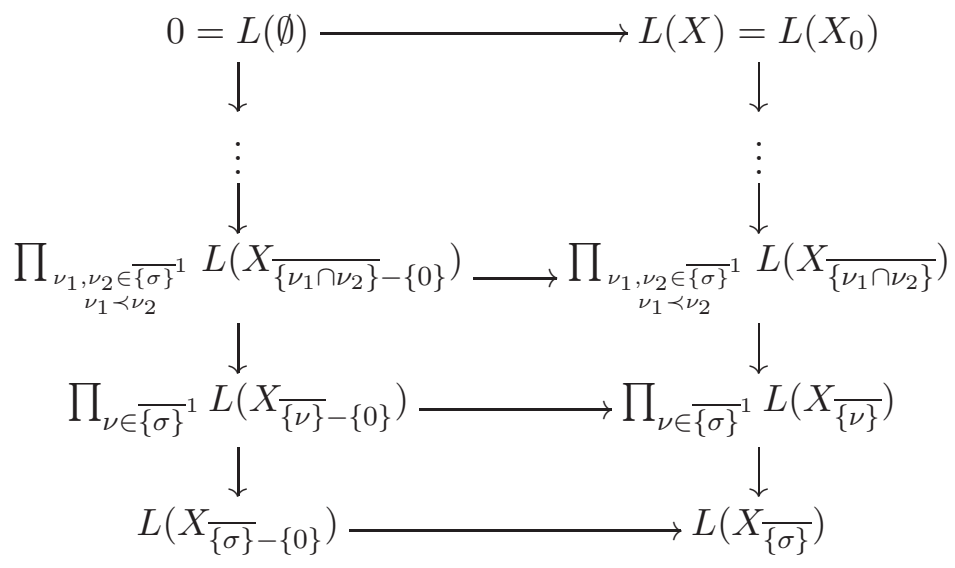

Again, we have total degree zero for $L\left(X_{\overline{\{\sigma\}}}\right)$, hence degree $-r$ for $L(X)$. We obtain the following lemma.

Lemma 4.8. Fix an ordering of $\{\nu \in \mathfrak{S} \mid \operatorname{dim} \nu=1, \nu$ face of $\sigma\}$.

(a) There is a canonical morphism of complexes of Nisnevich sheaves

$$
M V_{\sigma}: L\left(\mathfrak{Y}_{\sigma}^{\prime}\right) \longrightarrow L\left(\mathfrak{Y}_{\sigma}\right) .
$$

It is a quasi-isomorphism, and hence induces an isomorphism $\mathbf{R} C\left(M V_{\sigma}\right)$ in $D M_{-}^{\text {eff }}(k)$.

(b) The projection from $L\left(\mathfrak{Y}_{\sigma}^{\prime}\right)$ to its upper line

$$
p r_{\sigma}: L\left(\mathfrak{Y}_{\sigma}^{\prime}\right) \longrightarrow L(X)[\operatorname{dim} \sigma]
$$

induces an isomorphism $\mathbf{R} C\left(p r_{\sigma}\right)$ in $D M_{-}^{\text {eff }}(k)$.

Proof. Part (a) is the Mayer-Vietoris property, and part (b) is a consequence of Lemma 4.7.

Analyzing the dependence of the isomorphisms on the choice of the ordering, one finds the following.

Corollary 4.9. Let $\sigma \in \mathfrak{S}$. There is a canonical isomorphism

$$
M_{\mathrm{gm}}\left(X_{\sigma}, i_{\sigma}^{!} j ! \mathbb{Z}\right) \stackrel{\sim}{\longrightarrow} \operatorname{Hom}\left(\mathbb{Z}(\sigma), M_{\mathrm{gm}}(X)\right)[\operatorname{dim} \sigma]
$$

in $D M_{-}^{\text {eff }}(k)$. Any choice of an ordering $\prec$ as in Lemma 4.8 induces an isomorphism $\rho_{\prec}$ of the right-hand side with $M_{\mathrm{gm}}(X)[\operatorname{dim} \sigma]$. The induced isomorphism

$$
M_{\mathrm{gm}}\left(X_{\sigma}, i_{\sigma}^{!} j ! \mathbb{Z}\right) \stackrel{\sim}{\longrightarrow} M_{\mathrm{gm}}(X)[\operatorname{dim} \sigma]
$$

equals $\mathbf{R} C\left(p r_{\sigma}\right) \circ\left(\mathbf{R} C\left(M V_{\sigma}\right)\right)^{-1}$, where $p r_{\sigma}$ and $M V_{\sigma}$ are as in Lemma 4.8. 


\section{ON THE BOUNDARY MOTIVE OF A SHIMURA VARIETY}

We are ready for the proof of Theorem 4.4 (a) in the affine case, i.e. with $\mathfrak{S}$ replaced by $\overline{\{\tau\}}$, for $\tau \in \mathfrak{T}:$ recall that $M_{\mathrm{gm}}\left(X_{\mathfrak{T} \cap \overline{\{\tau\}}}, i_{\mathfrak{T}}^{!} j ! \mathbb{Z}\right)$ is associated to

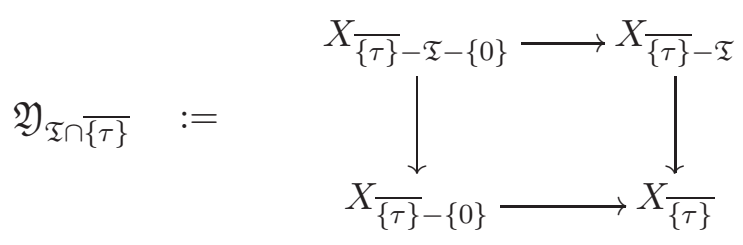

Lemma 4.10. Fix an ordering $\prec$ of $\{\nu \in \mathfrak{S} \mid \operatorname{dim} \nu=1, \nu$ face of $\tau\}$.

(a) There is a complex of Nisnevich sheaves $L\left(\mathfrak{Y}_{\mathfrak{T} \cap \overline{\{\tau\}}}^{\prime}\right)$, and a canonical quasi-isomorphism

$$
M V_{\mathfrak{T} \cap \overline{\{\tau\}}}: L\left(\mathfrak{Y}_{\mathfrak{T} \cap \overline{\{\tau\}}}^{\prime}\right) \longrightarrow L\left(\mathfrak{Y}_{\mathfrak{T} \cap \overline{\{\tau\}}}\right)
$$

(b) There is a morphism

$$
\rho_{\prec}^{-1} \circ p r_{\mathfrak{T} \cap \overline{\{\tau\}}}: L\left(\mathfrak{Y}_{\mathfrak{T} \cap \overline{\{\tau\}}}^{\prime}\right) \longrightarrow \operatorname{Hom}\left(C_{*}(\mathfrak{T} \cap \overline{\{\tau\}}, \mathbb{Z}), L(X)\right)
$$

inducing an isomorphism in $D M_{-}^{\text {eff }}(k)$.

(c) The resulting isomorphism

$$
M_{\mathrm{gm}}\left(X_{\mathfrak{T} \cap \overline{\{\tau\}}}, i_{\mathfrak{T}}^{!} j ! \mathbb{Z}\right) \stackrel{\sim}{\longrightarrow} \mathbf{R} C\left(\operatorname{Hom}\left(C_{*}(\mathfrak{T} \cap \overline{\{\tau\}}, \mathbb{Z}), L(X)\right)\right)
$$

in $D M_{-}^{\text {eff }}(k)$ does not depend on the choice of the ordering.

(d) If $\tau$ is a face of $\sigma \in \mathfrak{T}$, then any extension of $\prec$ to an ordering of $\{\nu \in \mathfrak{S} \mid \operatorname{dim} \nu=1$, $\nu$ face of $\sigma\}$ induces a canonical morphism of complexes

$$
L\left(\mathfrak{Y}_{\mathfrak{T} \cap \overline{\{\tau\}}}^{\prime}\right) \longrightarrow L\left(\mathfrak{Y}_{\mathfrak{T} \cap \overline{\{\sigma\}}}^{\prime}\right)
$$

such that

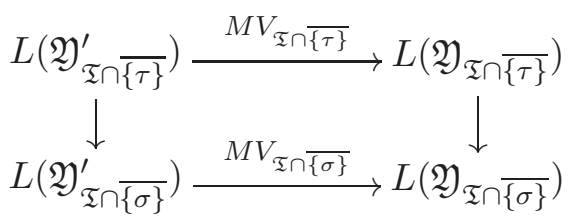

and

$$
\begin{aligned}
& L\left(\mathfrak{Y}_{\mathfrak{T} \cap \overline{\{\tau\}}}^{\prime}\right) \stackrel{\rho_{\prec}^{-1} \circ p r_{\mathfrak{T} \cap \overline{\{\tau\}}}}{\longrightarrow} \operatorname{Hom}\left(C_{*}(\mathfrak{T} \cap \overline{\{\tau\}}, \mathbb{Z}), L(X)\right) \\
& \qquad\left(\mathfrak{Y}_{\mathfrak{T} \cap \overline{\{\sigma\}}}^{\prime}\right) \stackrel{\rho_{\prec}^{-1} \circ p r_{\mathfrak{T} \cap \overline{\{\sigma\}}}}{\longrightarrow} \operatorname{Hom}\left(C _ { * } \left(\mathfrak{T} \cap \overline{\left.\left.\frac{\downarrow}{\{\sigma\}}, \mathbb{Z}\right), L(X)\right)}\right.\right.
\end{aligned}
$$

commute. Here, the morphism $\mathfrak{Y}_{\mathfrak{T} \cap \overline{\{\tau\}}} \longrightarrow \mathfrak{Y}_{\mathfrak{T} \cap \overline{\{\sigma\}}}$ is the natural morphism given by the inclusion $\iota$ of $\overline{\{\tau\}}$ into $\overline{\{\sigma\}}$, and

$$
\operatorname{Hom}\left(C_{*}(\mathfrak{T} \cap \overline{\{\tau\}}, \mathbb{Z}), L(X)\right) \longrightarrow \operatorname{Hom}\left(C_{*}(\mathfrak{T} \cap \overline{\{\sigma\}}, \mathbb{Z}), L(X)\right)
$$

is induced by the morphism $\iota^{*}$ from Remark 4.2 .

Proof. The lower row of $\mathfrak{Y}_{\mathfrak{T} \cap \overline{\{\tau\}}}$ contributes nothing to the motive $M_{\mathrm{gm}}\left(X_{\mathfrak{T} \cap \overline{\{\tau\}}}, i_{\mathfrak{T}}^{!} j ! \mathbb{Z}\right)$ thanks to Lemma 4.7 (note that $\tau \neq 0$ since $\tau \in \mathfrak{T}$ ). For the upper row

$$
\mathfrak{Z}_{\mathfrak{T} \cap \overline{\{\tau\}}}:=X_{\overline{\{\tau\}}-\mathfrak{T}-\{0\}} \longrightarrow X_{\overline{\{\tau\}}-\mathfrak{T}},
$$

we shall construct a double complex $L\left(\mathfrak{Z}_{\mathfrak{T} \cap \overline{\{\tau\}}}^{\prime}\right)$ of vertical length $\operatorname{dim} \tau$, and resolving $L\left(\mathfrak{Z}_{\mathfrak{T} \cap \overline{\{\tau\}}}\right)$ as in the construction preceding Lemma 4.8. Every row in $L\left(\mathfrak{Z}_{\mathfrak{T} \cap \overline{\{\tau\}}}^{\prime}\right)$ will be the direct product of one 


\section{J. Wildeshaus}

of two types of factors: either the factor is simply $0=L(\emptyset) \longrightarrow L(X)=L\left(X_{0}\right)$, or it has the shape $L\left(X_{\overline{\{\eta\}}-\{0\}}\right) \longrightarrow L\left(X_{\overline{\{\eta\}}}\right)$, for a face $\eta$ of $\tau$ not belonging to $\mathfrak{T}$, and not equal to zero. The vertical differentials of $L\left(\mathfrak{Z}_{\left.\mathfrak{T} \cap \frac{\prime}{\{\tau\}}\right)}^{\prime}\right.$ will be induced by the inclusions of faces.

For the construction of $L\left(\mathfrak{Z}_{\mathfrak{T} \cap \overline{\{\tau\}}}^{\prime}\right)$, we apply induction on the cardinality of $\mathfrak{T} \cap \overline{\{\tau\}}$, starting with Lemma 4.8 and Corollary 4.9 if $\mathfrak{T} \cap \overline{\{\tau\}}=\{\tau\}$. In the general case, consider the finite covering

$$
X_{\overline{\{\tau\}}-\mathfrak{T}}=\bigcup_{\nu \in \overline{\{\tau\}}^{1}} X_{\overline{\{\nu\}}-\mathfrak{T}},
$$

and observe that for each $\nu \in \overline{\{\tau\}}^{1}$, the cone $\tau$ is not contained in the set $\mathfrak{T} \cap \overline{\{\nu\}}$. Hence, the latter is properly contained in $\mathfrak{T} \cap \overline{\{\tau\}}$, and we may apply the induction step on each $X_{\overline{\{\eta\}}-\mathfrak{T}-\{0\}} \longrightarrow$ $X_{\overline{\{\eta\}}-\mathfrak{T}}$, for any intersection $\eta$ of cones in $\overline{\{\tau\}}^{1}$ contained in $\mathfrak{T}$. Note that for $\eta \notin \mathfrak{T}$, this row equals $X_{\overline{\{\eta\}}-\{0\}} \longrightarrow X_{\overline{\{\eta\}}}$. The result is the double complex $L\left(\mathfrak{Z}_{\mathfrak{T} \cap \overline{\{\tau\}}}^{\prime}\right)$. Note that the top row of $L\left(\mathfrak{Z}_{\mathfrak{T} \cap \overline{\{\tau\}}}^{\prime}\right)$ equals (one copy of) $0 \longrightarrow L(X)$. By definition, we get $L\left(\mathfrak{Y}_{\mathfrak{T} \cap \overline{\{\tau\}}}^{\prime}\right)$ by adding the lower row of $L\left(\mathfrak{Y}_{\mathfrak{T} \cap \overline{\{\tau\}}}\right)$. The canonical map from $L\left(\mathfrak{Y}_{\mathfrak{T} \cap \overline{\{\tau\}}}^{\prime}\right)$ to $L\left(\mathfrak{Y}_{\mathfrak{T} \cap \overline{\{\tau\}}}\right)$ is a quasi-isomorphism thanks to the Mayer-Vietoris property. The factors of the shape $L\left(X_{\overline{\{\eta\}}-\{0\}}\right) \longrightarrow L\left(X_{\overline{\{\eta\}}}\right)$, with $\eta \neq 0$, organize into a sub-complex of $L\left(\mathfrak{Y}_{\mathfrak{T} \cap \overline{\{\tau\}}}^{\prime}\right)$. (Note that each such $\eta$ is either a face of $\tau$ not belonging to $\mathfrak{T}$, or equal to $\tau$, in which case the factor in question is the last row of $L\left(\mathfrak{Y}_{\mathfrak{T} \cap \overline{\{\tau\}}}^{\prime}\right)$.) By Lemma 4.7, this sub-complex vanishes in $D M_{-}^{\text {eff }}(k)$. Furthermore, by induction, the quotient of $L\left(\mathfrak{Y}_{\mathfrak{T} \cap \overline{\{\tau\}}}^{\prime}\right)$ by this subcomplex consists of the top row $0 \longrightarrow L(X)$ of $L\left(\mathfrak{Y}_{\mathfrak{T} \cap \overline{\{\tau\}}}^{\prime}\right)$, and the $\operatorname{Hom}\left(C_{*}(\mathfrak{T} \cap \overline{\{\eta\}}, \mathbb{Z}), L(X)\right)$, for all intersections $\eta$ of cones in $\overline{\{\tau\}}^{1}$ contained in $\mathfrak{T}$. More precisely, this quotient equals $\operatorname{Hom}\left(C_{*}^{\prime}(\mathfrak{T} \cap\right.$ $\overline{\{\tau\}}, \mathbb{Z}), L(X))$, where $C_{*}^{\prime}(\mathfrak{T} \cap \overline{\{\tau\}}, \mathbb{Z})$ is the complex defined as follows: for $m>-\operatorname{dim} \tau$, we have

$$
C_{m}^{\prime}(\mathfrak{T} \cap \overline{\{\tau\}}, \mathbb{Z}):=\bigoplus_{p+q=m} \bigoplus_{\eta} C_{p}(\mathfrak{T} \cap \overline{\{\eta\}}, \mathbb{Z})
$$

where $\eta$ runs through all $(-q+1)$-fold intersections of cones in $\overline{\{\tau\}}^{1}$. We have $C_{-\operatorname{dim} \tau}^{\prime}(\mathfrak{T} \cap \overline{\{\tau\}}, \mathbb{Z}):=$ $\mathbb{Z}(\tau)$, and $C_{m}^{\prime}(\mathfrak{T} \cap \overline{\{\tau\}}, \mathbb{Z}):=0$ for $m \notin[-\operatorname{dim} \tau, 0]$. The differential $C_{-(\operatorname{dim} \tau-1)}^{\prime}(\mathfrak{T} \cap \overline{\{\tau\}}, \mathbb{Z}) \longrightarrow$ $C_{-\operatorname{dim} \tau}^{\prime}(\mathfrak{T} \cap \overline{\{\tau\}}, \mathbb{Z})$ is the direct sum of maps $\mathbb{Z}(\eta) \rightarrow \mathbb{Z}(\tau)$, for faces $\eta$ of $\tau$. These maps are all isomorphisms. On orientations, they are described by adding the missing faces of dimension one, in the fixed order. It remains to construct a morphism $i: C_{*}(\mathfrak{T} \cap \overline{\{\tau\}}, \mathbb{Z}) \longrightarrow C_{*}^{\prime}(\mathfrak{T} \cap \overline{\{\tau\}}, \mathbb{Z})$. In degree $-\operatorname{dim} \tau$, we take the identity on $\mathbb{Z}(\tau)$. In degree $m>-\operatorname{dim} \tau$, take $\sigma \in \mathfrak{T} \cap \overline{\{\tau\}}$ of dimension $-m$, so $\mathbb{Z}(\sigma)$ is a summand of $C_{m}(\mathfrak{T} \cap \overline{\{\tau\}}, \mathbb{Z})$. It is also a summand of $C_{m}(\mathfrak{T} \cap \overline{\{\eta\}}, \mathbb{Z})$, for any face $\eta$ of $\tau$ containing $\sigma$ as a face, and we define $i_{m}$ on $\mathbb{Z}(\sigma)$ as the diagonal embedding of $\mathbb{Z}(\sigma)$ in $\bigoplus_{\eta} C_{m}(\mathfrak{T} \cap \overline{\{\eta\}}, \mathbb{Z}) \subset C_{m}^{\prime}(\mathfrak{T} \cap \overline{\{\tau\}}, \mathbb{Z})$. We leave it to the reader to check that this defines a morphism of complexes and, in fact, a quasi-isomorphism. Since both $C_{*}(\mathfrak{T} \cap \overline{\{\tau\}}, \mathbb{Z})$ and $C_{*}^{\prime}(\mathfrak{T} \cap \overline{\{\tau\}}, \mathbb{Z})$ are bounded complexes of free Abelian groups, it follows that $i$ is even a homotopy equivalence. Hence, the induced morphism

$$
\operatorname{Hom}\left(C_{*}^{\prime}(\mathfrak{T} \cap \overline{\{\eta\}}, \mathbb{Z}), L(X)\right) \longrightarrow \operatorname{Hom}\left(C_{*}(\mathfrak{T} \cap \overline{\{\eta\}}, \mathbb{Z}), L(X)\right)
$$

is a homotopy equivalence as well.

Proof of Theorem 4.4. By Proposition 4.1(b), the system $\mathfrak{U}_{X_{\mathfrak{S}}}$ given as $\left(X_{\overline{\{\tau\}}}\right)_{\tau \in \mathfrak{T}}$ lies in $\operatorname{Cov}^{(\infty)}\left(X_{\mathfrak{S}}\right)_{X_{\mathfrak{T}}}$. By Definition 2.9 and the remark following it, the completed motive $M_{\mathrm{gm}}^{\wedge}\left(X_{\mathfrak{T}}, i_{\mathfrak{T}}^{!} j ! \mathbb{Z}\right)$ 


\section{On the Boundary motive of a SHIMURA VARIETy}

is represented by the simple complex associated to $\underline{C}_{*}\left(L^{\bullet}\left(\mathfrak{U}_{\mathfrak{Y}}\right)\right)$, with

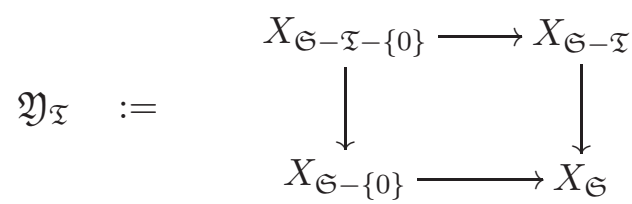

Recall that $L^{\bullet}\left(\mathfrak{U}_{\mathfrak{Y}}\right)$ is the simple complex associated to

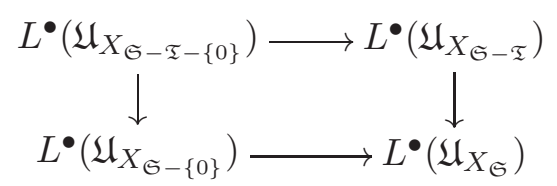

with $L^{\bullet}\left(\mathfrak{U}_{X_{\mathfrak{S}}}\right)$ sitting in degree $(0, *)$. We may represent $L^{\bullet}\left(\mathfrak{U}_{\mathfrak{Y}}\right)$ as follows: define a triple complex whose term $(-m, *, *)$ is the direct product of the $L\left(\mathfrak{Y}_{\mathfrak{T} \cap \overline{\{\tau\}}}\right)$, for all $(m+1)$-fold intersections $\tau$ in $\mathfrak{T}$. Then $L^{\bullet}\left(\mathfrak{U}_{\mathfrak{Y} \mathfrak{T}}\right)$ is the simple complex associated to this triple complex. By Lemma $4.10, L^{\bullet}\left(\mathfrak{U}_{\mathfrak{Y}}\right)$ may be replaced by the simple complex $s L^{\bullet, \bullet}$ associated to $L^{\bullet \bullet \bullet}$, with

$$
L^{p, q}:=\prod_{\tau} \operatorname{Hom}\left(C_{p}(\mathfrak{T} \cap \overline{\{\tau\}}, \mathbb{Z}), L(X)\right),
$$

where $\tau$ runs through all $(-q+1)$-fold intersections in $\mathfrak{T}$. Note that $L^{p, q}$ is non-zero only if $p$ and $q$ are non-positive.

We need to define a morphism

$$
s L^{\bullet, \bullet} \longrightarrow \operatorname{Hom}\left(C_{*}(\mathfrak{T}, \mathbb{Z}), L(X)\right),
$$

or in other words, a family of morphisms

$$
\prod_{p+q=m} L^{p, q} \longrightarrow \operatorname{Hom}\left(C_{m}(\mathfrak{T}, \mathbb{Z}), L(X)\right)
$$

compatible with the differentials. We shall define the dual morphisms

$$
i_{m}: C_{m}(\mathfrak{T}, \mathbb{Z}) \longrightarrow \bigoplus_{p+q=m} \bigoplus_{\tau} C_{p}(\mathfrak{T} \cap \overline{\{\tau\}}, \mathbb{Z}) .
$$

Let $\eta \in \mathfrak{T}$ be of dimension $-m$, so $\mathbb{Z}(\eta)$ is a summand of $C_{m}(\mathfrak{T}, \mathbb{Z})$. It is also a summand of $C_{m}(\mathfrak{T} \cap \overline{\{\tau\}}, \mathbb{Z})$, for any $\tau$ containing $\eta$ as a face, and we define $i_{m}$ on $\mathbb{Z}(\eta)$ as the diagonal embedding of $\mathbb{Z}(\eta)$ in $\bigoplus_{\tau} C_{m}(\mathfrak{T} \cap \overline{\{\tau\}}, \mathbb{Z})$, where the direct sum extends over all (finitely many!) $\tau$ containing $\eta$ as a face. This defines the morphism of complexes

$$
i: C_{*}(\mathfrak{T}, \mathbb{Z}) \longrightarrow s C_{*, *}(\mathfrak{T}, \mathbb{Z}),
$$

where the right-hand side denotes the simple complex associated to $C_{*, *}(\mathfrak{T}, \mathbb{Z})$, with

$$
C_{p, q}(\mathfrak{T}, \mathbb{Z}):=\bigoplus_{\tau} C_{p}(\mathfrak{T} \cap \overline{\{\tau\}}, \mathbb{Z})
$$

as above. One sees that $i$ factors through $Z_{*, 0}(\mathfrak{T}, \mathbb{Z}) \subset s C_{*, *}(\mathfrak{T}, \mathbb{Z})$, with $Z_{p, q}(\mathfrak{T}, \mathbb{Z})$ denoting the kernel of the differential in $q$-direction

$$
d_{q}: C_{p, q}(\mathfrak{T}, \mathbb{Z}) \longrightarrow C_{p, q-1}(\mathfrak{T}, \mathbb{Z}) .
$$

Given the definition of that differential, it is not difficult to check that the complex

$$
C_{m}(\mathfrak{T}, \mathbb{Z}) \stackrel{i_{m}}{\longrightarrow} C_{m, 0}(\mathfrak{T}, \mathbb{Z}) \stackrel{d_{0}}{\longrightarrow} C_{m,-1}(\mathfrak{T}, \mathbb{Z}) \stackrel{d_{-1}}{\longrightarrow} \cdots
$$

is acyclic for every $m$. The complex $C_{*}(\mathfrak{T}, \mathbb{Z})$ is bounded, hence $i$ is a quasi-isomorphism. Since both $C_{*}(\mathfrak{T}, \mathbb{Z})$ and $s C_{*, *}(\mathfrak{T}, \mathbb{Z})$ are complexes of free Abelian groups, and $C_{*}(\mathfrak{T}, \mathbb{Z})$ is bounded, it follows 


\section{J. Wildeshaus}

that $i$ is even a homotopy equivalence. Hence, the induced morphism

$$
s L^{\bullet \bullet \bullet}=\operatorname{Hom}\left(s C_{*, *}(\mathfrak{T}, \mathbb{Z}), L(X)\right) \longrightarrow \operatorname{Hom}\left(C_{*}(\mathfrak{T}, \mathbb{Z}), L(X)\right)
$$

is a homotopy equivalence as well.

Example 4.11. Note that the conclusion of Theorem 4.4(c) is false, in general, if $D$ is only contractible, but not open. As an example, take $\mathfrak{T}$ to consist of two cones: $\sigma$, of dimension at least two, and (only) one of the faces of $\sigma$ of codimension one. The conclusion of Theorem 4.4(c) is false since $M_{\mathrm{gm}}\left(X_{\mathfrak{T}}, i_{\mathfrak{T}}^{!} j ! \mathbb{Z}\right)=0$ by Theorem $4.4(\mathrm{~b})$.

\section{Notation for Shimura varieties}

We recall the notation for Shimura varieties and their compactifications. It is identical to that used in [Pin90, Pin92, Wil00, BW04], except that we change the capital letter $M$ used to denote Shimura varieties in those references to capital $S$ in order to avoid confusion with the motivic notation introduced earlier in this paper.

Let $(P, \mathfrak{X})$ be mixed Shimura data [Pin90, Definition 2.1]. In particular, $P$ is a connected algebraic linear group over $\mathbb{Q}$, and $P(\mathbb{R})$ acts on the complex manifold $\mathfrak{X}$ by analytic automorphisms. Denote by $W$ the unipotent radical of $P$. If $P$ is reductive, i.e. if $W=0$, then $(P, \mathfrak{X})$ is called pure. The Shimura varieties associated to $(P, \mathfrak{X})$ are indexed by the open compact subgroups of $P\left(\mathbb{A}_{f}\right)$. If $K$ is one such group, then the analytic space of $\mathbb{C}$-valued points of the corresponding variety is given as $S^{K}(\mathbb{C}):=P(\mathbb{Q}) \backslash\left(\mathfrak{X} \times P\left(\mathbb{A}_{f}\right) / K\right)$, where $\mathbb{A}_{f}$ denotes the ring of finite adèles over $\mathbb{Q}$. According to Pink's generalization to mixed Shimura varieties of the algebraization theorem of Baily and Borel [Pin90, Proposition 9.24], there exist canonical structures of normal algebraic varieties on the $S^{K}(\mathbb{C})$, which we denote as $S_{\mathbb{C}}^{K}:=S^{K}(P, \mathfrak{X})_{\mathbb{C}}$. According to [Moo98, Theorem 2.18] and [Pin90, Theorem 11.18], there is a canonical model of $S_{\mathbb{C}}^{K}$, which we denote as $S^{K}:=S^{K}(P, \mathfrak{X})$. It is defined over the reflex field $E(P, \mathfrak{X})$ of $(P, \mathfrak{X})$ (see [Pin90, §11.1]).

Any admissible parabolic subgroup (see [Pin90, Definition 4.5]) $Q$ of $P$ has a canonical normal subgroup $P_{1}$ (see $[$ Pin90, $\S 4.7]$ ). There is a finite collection of rational boundary components $\left(P_{1}, \mathfrak{X}_{1}\right)$, indexed by the $P_{1}(\mathbb{R})$-orbits in $\pi_{0}(\mathfrak{X})$ (see $\left.[\operatorname{Pin} 90, \S 4.11]\right)$. The $\left(P_{1}, \mathfrak{X}_{1}\right)$ are themselves mixed Shimura data. Consider the following condition on $(P, \mathfrak{X})$.

(+) If $G$ denotes the maximal reductive quotient of $P$, then the neutral connected component $Z(G)^{0}$ of the center $Z(G)$ of $G$ is, up to isogeny, a direct product of a $\mathbb{Q}$-split torus with a torus $T$ of compact type (i.e. $T(\mathbb{R})$ is compact) defined over $\mathbb{Q}$.

If $(P, \mathfrak{X})$ satisfies $(+)$, then so does any rational boundary component $\left(P_{1}, \mathfrak{X}_{1}\right)$ (see [Pin90, Proof of Corollary 4.10]). Denote by $U_{1} \subset P_{1}$ the 'weight -2 ' part of $P_{1}$. It is Abelian, normal in $Q$, and central in the unipotent radical $W_{1}$ of $P_{1}$. Fix a connected component $\mathfrak{X}^{0}$ of $\mathfrak{X}$, and denote by $\left(P_{1}, \mathfrak{X}_{1}\right)$ the associated rational boundary component. There is a natural open embedding $\iota: \mathfrak{X}^{0} \longleftrightarrow \mathfrak{X}_{1}$ (see [Pin90, $\S 4.11$, Proposition 4.15(a)]). If $\mathfrak{X}_{1}^{0}$ denotes the connected component of $\mathfrak{X}_{1}$ containing $\mathfrak{X}^{0}$, then the image of the embedding can be described by means of the map imaginary part

$$
\operatorname{im}: \mathfrak{X}_{1} \longrightarrow U_{1}(\mathbb{R})(-1):=\frac{1}{2 \pi i} \cdot U_{1}(\mathbb{R}) \subset U_{1}(\mathbb{C})
$$

of $[$ Pin90, $\S 4.14]: \iota\left(\mathfrak{X}^{0}\right)$ is the pre-image of an open convex cone $C\left(\mathfrak{X}^{0}, P_{1}\right) \subset U_{1}(\mathbb{R})(-1)$ under im $\left.\right|_{\mathfrak{X}_{1}^{0}}$ (see [Pin90, Proposition 4.15(b)]). The reflex field does not change when passing from $(P, \mathfrak{X})$ to a rational boundary component [Pin90, Proposition 12.1]. 


\section{ON THE BOUNDARY MOTIVE OF A SHIMURA VARIETY}

\section{Toroidal compactifications of Shimura varieties}

In order to discuss toroidal compactifications, we need to introduce the conical complex associated to $(P, \mathfrak{X})$ : set-theoretically, it is defined as

$$
\mathcal{C}(P, \mathfrak{X}):=\coprod_{\left(\mathfrak{X}^{0}, P_{1}\right)} C\left(\mathfrak{X}^{0}, P_{1}\right) .
$$

By [Pin90, §4.24], the conical complex is naturally equipped with a topology (usually different from the coproduct topology). The closure $C^{*}\left(\mathfrak{X}^{0}, P_{1}\right)$ of $C\left(\mathfrak{X}^{0}, P_{1}\right)$ inside $\mathcal{C}(P, \mathfrak{X})$ can still be considered as a convex cone in $U_{1}(\mathbb{R})(-1)$, with the induced topology.

For a fixed open compact subgroup $K \subset P\left(\mathbb{A}_{f}\right)$, the (partial) toroidal compactifications of $S^{K}$ are parameterized by $K$-admissible partial cone decompositions, which are collections of subsets of $\mathcal{C}(P, \mathfrak{X}) \times P\left(\mathbb{A}_{f}\right)$ satisfying the axioms of $[\operatorname{Pin} 90, \S 6.4]$. If $\mathfrak{S}$ is one such, then, in particular, any member of $\mathfrak{S}$ is of the shape $\sigma \times\{p\}, p \in P\left(\mathbb{A}_{f}\right), \sigma \subset C^{*}\left(\mathfrak{X}^{0}, P_{1}\right)$ a convex rational polyhedral cone in the vector space $U_{1}(\mathbb{R})(-1)$ not containing any non-trivial linear subspace.

Let $S^{K}(\mathfrak{S}):=S^{K}(P, \mathfrak{X}, \mathfrak{S})$ be the associated compactification; we refer to [Pin90, SS9.27 and 9.28] for criteria sufficient to guarantee its existence. It comes equipped with a natural stratification into locally closed strata. The unique open stratum is $S^{K}$. Any stratum different from the generic stratum is obtained as follows. Fix a pair $\left(\mathfrak{X}^{0}, P_{1}\right)$ as above, $p \in P\left(\mathbb{A}_{f}\right)$ and $\sigma \times\{p\} \in \mathfrak{S}$ such that $\sigma \subset C^{*}\left(\mathfrak{X}^{0}, P_{1}\right), \sigma \cap C\left(\mathfrak{X}^{0}, P_{1}\right) \neq \emptyset$, and the admissible parabolic subgroup $Q$ giving rise to $P_{1}$ is proper, i.e. not equal to $P$. To $\sigma$, one associates Shimura data $\left(P_{1,[\sigma]}, \mathfrak{X}_{1,[\sigma]}\right)$ (see $[$ Pin90, $\S 7.1]$ ), whose underlying group $P_{1,[\sigma]}$ is the quotient of $P_{1}$ by the algebraic subgroup $\langle\sigma\rangle \subset U_{1}$ satisfying $\mathbb{R} \cdot \sigma=1 / 2 \pi i \cdot\langle\sigma\rangle(\mathbb{R})$. Set

$$
K_{1}:=P_{1}\left(\mathbb{A}_{f}\right) \cap p \cdot K \cdot p^{-1}, \quad \pi_{[\sigma]}: P_{1} \rightarrow P_{1,[\sigma]} .
$$

According to $[$ Pin90, $\S 7.3]$, there is a canonical map

$$
i_{\sigma, p}(\mathbb{C}): S^{\pi_{[\sigma]}\left(K_{1}\right)}\left(P_{1,[\sigma]}, \mathfrak{X}_{1,[\sigma]}\right)(\mathbb{C}) \longrightarrow S^{K}(\mathfrak{S})(\mathbb{C}):=S^{K}(P, \mathfrak{X}, \mathfrak{S})(\mathbb{C})
$$

whose image is locally closed. In fact, the source and the target of $i_{\sigma, p}(\mathbb{C})$ have canonical models over the reflex field $E(P, \mathfrak{X})$, and $i_{\sigma, p}(\mathbb{C})$ comes from a morphism of schemes over $E(P, \mathfrak{X})$ (see [Pin90, Theorem 12.4]) denoted $i_{\sigma, p}$. If $(P, \mathfrak{X})$ satisfies $(+)$, and $K$ is neat (see, e.g., [Pin90, $\left.\left.\S 0.6\right]\right)$, then $i_{\sigma, p}$ is an immersion, i.e. it identifies $S^{\pi_{[\sigma]}\left(K_{1}\right)}$ with a locally closed sub-scheme of $M^{K}(\mathfrak{S})$ (see [Wil00, Proposition 1.6]). Denote by $j: S^{K} \longleftrightarrow S^{K}(\mathfrak{S})$ the open immersion, and by $S^{K_{1}}$ the Shimura variety $S^{K_{1}}\left(P_{1}, \mathfrak{X}_{1}\right)$. Recall the definition of $M_{\mathrm{gm}}\left(S^{\pi_{[\sigma]}\left(K_{1}\right)}, i_{\sigma, p}^{!} j ! \mathbb{Z}\right)$, the motive of $S^{\pi_{[\sigma]}\left(K_{1}\right)}$ with coefficients in $i_{\sigma, p}^{!} j ! \mathbb{Z}$ (see [Wil06, Definition 3.1]), and of the group of orientations $\mathbb{Z}(\sigma)$ of $\sigma$ (see $\S 4$ ). We now give our main result concerning strata in toroidal compactifications.

Theorem 6.1. Assume that $(P, \mathfrak{X})$ satisfies $(+)$, and that $K$ is neat. Then there is a canonical isomorphism

$$
M_{\mathrm{gm}}\left(S^{\pi_{[\sigma]}\left(K_{1}\right)}, i_{\sigma, p} j ! \mathbb{Z}\right) \stackrel{\sim}{\longrightarrow} \operatorname{Hom}\left(\mathbb{Z}(\sigma), M_{\mathrm{gm}}\left(S^{K_{1}}\right)\right)[\operatorname{dim} \sigma]
$$

in $D M_{-}^{\text {eff }}(E(P, \mathfrak{X}))$.

Proof. Let $\mathfrak{S}_{1,[\sigma]}$ be the minimal $K_{1}$-admissible cone decomposition of $\mathcal{C}\left(P_{1}, \mathfrak{X}_{1}\right) \times P_{1}\left(\mathbb{A}_{f}\right)$ containing $\sigma \times\{1\}$. It can be realized inside the decomposition $\mathfrak{S}_{1}^{0}$ of [Pin90, $\left.\S 6.13\right]$; by definition, $\mathfrak{S}_{1,[\sigma]}$ is concentrated in the unipotent fibre [Pin90, $\S 6.5(\mathrm{~d})]$. View $S^{\pi_{[\sigma]}\left(K_{1}\right)}$ as sitting inside $S^{K_{1}}\left(\mathfrak{S}_{1,[\sigma]}\right)$ : $i_{1}: S^{\pi_{[\sigma]}\left(K_{1}\right)} \hookrightarrow S^{K_{1}}\left(\mathfrak{S}_{1,[\sigma]}\right)$. In fact, $j_{1}: S^{K_{1}} \hookrightarrow S^{K_{1}}\left(\mathfrak{S}_{1,[\sigma]}\right)$ is a relative affine torus embedding over $S^{\pi_{[\sigma]}\left(K_{1}\right)}$, and $i_{1}$ identifies $S^{\pi_{[\sigma]}\left(K_{1}\right)}$ with the unique closed stratum of the canonical 


\section{J. WiLdeshaus}

stratification [Wil00, Proposition 1.15 and Lemma 1.16]. Consider the diagram

$$
S^{\pi_{[\sigma]}\left(K_{1}\right) \stackrel{i_{\sigma, p}}{\longrightarrow} S^{K}(\mathfrak{S})}{ }_{i^{K_{1}}}\left(\mathfrak{S}_{1,[\sigma]}\right)
$$

According to [Wil00, Theorem 1.13], there is a canonical isomorphism

$$
f:\left(S^{K}(\mathfrak{S})\right)_{S^{\pi}[\sigma]}\left(K_{1}\right) \stackrel{\sim}{\longrightarrow}\left(S^{K_{1}}\left(\mathfrak{S}_{1,[\sigma]}\right)\right)_{S^{\pi}[\sigma]}\left(K_{1}\right)
$$

between the formal completions of $S^{K}(\mathfrak{S})$ and of $S^{K_{1}}\left(\mathfrak{S}_{1,[\sigma]}\right)$ along $S^{\pi_{[\sigma]}\left(K_{1}\right)}$ compatible with the immersions $i_{\sigma, p}$ and $i_{1}$. By analytical invariance [Wil06, Theorem 5.1], $f$ induces an isomorphism

$$
M_{\mathrm{gm}}\left(S^{\pi_{[\sigma]}\left(K_{1}\right)}, i_{\sigma, p}^{!} j_{!} \mathbb{Z}\right) \stackrel{\sim}{\longrightarrow} M_{\mathrm{gm}}\left(S^{\pi_{[\sigma]}\left(K_{1}\right)}, i_{1}^{!} j_{1 !} \mathbb{Z}\right) .
$$

Now apply Corollary 4.9 .

Note [Wil06, Remark 3.2] that the left-hand side of the isomorphism from Theorem 6.1 lies in the full triangulated sub-category $D M_{\mathrm{gm}}^{\mathrm{eff}}(E(P, \mathfrak{X}))$ of $D M_{-}^{\mathrm{eff}}(E(P, \mathfrak{X}))$, and hence in the category $D M_{\mathrm{gm}}(E(P, \mathfrak{X}))$ of geometrical motives over $E(P, \mathfrak{X})$. This latter category is a rigid tensor category [Voe00, Theorem 4.3.7, 1. and 2.]. In particular, there exists an internal Hom functor

$$
\underline{H o m}: D M_{\mathrm{gm}}(E(P, \mathfrak{X})) \times D M_{\mathrm{gm}}(E(P, \mathfrak{X})) \longrightarrow D M_{\mathrm{gm}}(E(P, \mathfrak{X})) .
$$

Writing $M^{*}:=\underline{\operatorname{Hom}}(M, \mathbb{Z}(0))$, we thus have $M=\left(M^{*}\right)^{*}$ for all $M \in D M_{\mathrm{gm}}(E(P, \mathfrak{X}))$.

If $K$ is neat, then $S^{K}$ is in $S m / E(P, \mathfrak{X})$. Recall that in this case, the motive with compact support of $S^{\pi_{[\sigma]}\left(K_{1}\right)}$ and with coefficients in $i_{\sigma, p}^{*} j_{*} \mathbb{Z}$ is defined as

$$
M_{\mathrm{gm}}^{c}\left(S^{\pi_{[\sigma]}\left(K_{1}\right)}, i_{\sigma, p}^{*} j_{*} \mathbb{Z}\right):=M_{\mathrm{gm}}\left(S^{\pi_{[\sigma]}\left(K_{1}\right)}, i_{\sigma, p}^{!} j ! \mathbb{Z}\right)^{*}\left(\operatorname{dim} S^{K}\right)\left[2 \operatorname{dim} S^{K}\right]
$$

(see [Wil06, Definition 7.1]). It lies in the full triangulated sub-category $D M_{\mathrm{gm}}^{\mathrm{eff}}(E(P, \mathfrak{X}))$ of $D M_{\mathrm{gm}}(E(P, \mathfrak{X}))$.

Corollary 6.2. Assume that $(P, \mathfrak{X})$ satisfies $(+)$, and that $K$ is neat. Then there is a canonical isomorphism

$$
M_{\mathrm{gm}}^{c}\left(S^{\pi_{[\sigma]}\left(K_{1}\right)}, i_{\sigma, p}^{*} j_{*} \mathbb{Z}\right) \stackrel{\sim}{\longrightarrow} \operatorname{Hom}\left(\mathbb{Z}(\sigma), M_{\mathrm{gm}}^{c}\left(S^{K_{1}}\right)\right)[-\operatorname{dim} \sigma]
$$

in $D M_{\mathrm{gm}}^{\mathrm{eff}}(E(P, \mathfrak{X}))$.

Proof. Since the group $\mathbb{Z}(\sigma)$ is canonically self-dual, Theorem 6.1 tells us that $M_{\mathrm{gm}}^{c}\left(S^{\pi_{[\sigma]}\left(K_{1}\right)}, i_{\sigma, p}^{*} j_{*} \mathbb{Z}\right)$ is canonically isomorphic to

$$
\operatorname{Hom}\left(\mathbb{Z}(\sigma), M_{\mathrm{gm}}\left(S^{K_{1}}\right)^{*}\left(\operatorname{dim} S^{K}\right)\right)\left[2 \operatorname{dim} S^{K}-\operatorname{dim} \sigma\right] .
$$

Note that $\operatorname{dim} S^{K}=\operatorname{dim} S^{K_{1}}$. According to [Voe00, Theorem 4.3.7, 3.], there is a canonical isomorphism

$$
M_{\mathrm{gm}}^{c}\left(S^{K_{1}}\right) \stackrel{\sim}{\longrightarrow} M_{\mathrm{gm}}\left(S^{K_{1}}\right)^{*}\left(\operatorname{dim} S^{K_{1}}\right)\left[2 \operatorname{dim} S^{K_{1}}\right] .
$$

This gives the desired isomorphism.

Theorem 6.1 and Corollary 6.2 identify the motives occurring as graded pieces in the colocalization [Wil06, §3] (respectively localization [Wil06, §7]) filtration of the boundary motive $\partial M_{\mathrm{gm}}\left(S^{K}\right)$ introduced in [Wil06], and associated to the stratification of a toroidal compactification. When $S^{K}$ is pure, the filtration associated to the Baily-Borel compactification to be discussed in the next section is usually more efficient. 


\section{ON THE BOUNDARY MOTIVE OF A SHIMURA VARIETY}

\section{The Baily-Borel compactification of pure Shimura varieties}

Assume that $(P, \mathfrak{X})=(G, \mathfrak{H})$ is pure. Fix an open compact subgroup $K \subset G\left(\mathbb{A}_{f}\right)$. Let $\left(S^{K}\right)^{*}$ denote the Baily-Borel compactification of $S^{K}$ [BB66, AMRT75]. It comes equipped with a natural stratification into locally closed strata. The unique open stratum is $S^{K}$. Any stratum different from the generic stratum is obtained as follows. Fix a proper admissible parabolic subgroup $Q$ of $G$ with associated normal subgroup $P_{1}$. Fix a rational boundary component $\left(P_{1}, \mathfrak{X}_{1}\right)$ of $(G, \mathfrak{H})$, and an element $g \in G\left(\mathbb{A}_{f}\right)$. Define $K^{\prime}:=g \cdot K \cdot g^{-1}$, and $K_{1}:=P_{1}\left(\mathbb{A}_{f}\right) \cap K^{\prime}$. Denote by $W_{1}$ the unipotent radical of $P_{1}$, and by $\pi:\left(P_{1}, \mathfrak{X}_{1}\right) \rightarrow\left(G_{1}, \mathfrak{H}_{1}\right):=\left(P_{1}, \mathfrak{X}_{1}\right) / W_{1}$ the quotient of $\left(P_{1}, \mathfrak{X}_{1}\right)$ by $W_{1}$ (see [Pin90, Proposition 2.9]). From the proof of [Pin90, Lemma 4.8], it follows that $W_{1}$ equals the unipotent radical of $Q$. According to [Pin90, $\S 7.6]$, there is a canonical morphism

$$
i_{g}: S^{\pi\left(K_{1}\right)}:=S^{\pi\left(K_{1}\right)}\left(G_{1}, \mathfrak{H}_{1}\right) \longrightarrow\left(S^{K}\right)^{*}
$$

whose image is locally closed, and identical to the stratum in question. Define the following group (cf. [BW04, § 1]):

$$
H_{Q}:=\underset{Q(\mathbb{Q})}{\operatorname{Stab}}\left(\mathfrak{H}_{1}\right) \cap P_{1}\left(\mathbb{A}_{f}\right) \cdot K^{\prime}
$$

Here, the symbol $\operatorname{Stab}_{Q(\mathbb{Q})}\left(\mathfrak{H}_{1}\right)$ denotes the subgroup of $Q(\mathbb{Q})$ stabilizing $\mathfrak{H}_{1} \cdot H_{Q}$ acts by analytic automorphisms on $\mathfrak{H}_{1} \times P_{1}\left(\mathbb{A}_{f}\right) / K_{1}$. Hence, the group $\Delta_{1}:=H_{Q} / P_{1}(\mathbb{Q})$ acts naturally on

$$
S^{\pi\left(K_{1}\right)}(\mathbb{C})=P_{1}(\mathbb{Q}) \backslash\left(\mathfrak{H}_{1} \times P_{1}\left(\mathbb{A}_{f}\right) / K_{1}\right) .
$$

This action is one by automorphisms of schemes over $E(G, \mathfrak{H})$ (see [Pin90, Proposition 9.24]). By $[$ Pin90, $\S 6.3]$, it factors through a finite quotient of $\Delta_{1}$. The quotient by this action is precisely the image of $i_{g}$ :

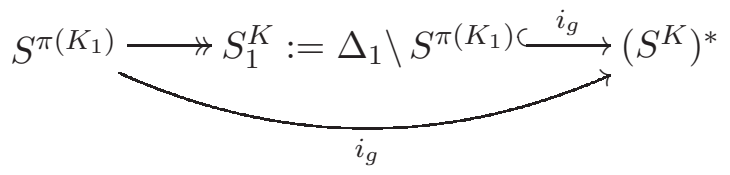

By abuse of notation, we denote by the same letter $i_{g}$ the inclusion of the stratum $S_{1}^{K}$ into $\left(S^{K}\right)^{*}$. Note that $\Delta_{1}$ is an arithmetic subgroup of $Q / P_{1}(\mathbb{Q})$, which is neat if $K$ is. By [BS73, $\left.\S 11.1(\mathrm{c})\right]$, such a group is of type $F L$, and hence satisfies condition (B) from $\S 3$. Denote by $j: S^{K} \longleftrightarrow\left(S^{K}\right)^{*}$ the open immersion, by $S^{K_{1}}$ the Shimura variety $S^{K_{1}}\left(P_{1}, \mathfrak{X}_{1}\right)$, by $u_{1}$ the dimension of the Abelian unipotent group $U_{1}$, and by $\mathbb{Z}\left(U_{1}(\mathbb{R})\right)$ the group of orientations of the vector space of its $\mathbb{R}$-valued points. Recall the definition of the $\Delta_{1}$-equivariant motive $M\left(S^{K_{1}}\right) \in \Delta_{1}-D M_{-}^{\text {eff }}(E(G, \mathfrak{H}))$, and of the functor

$$
R \Gamma\left(\Delta_{1}, \bullet\right): \Delta_{1}-D M_{-}^{\mathrm{eff}}(E(G, \mathfrak{H})) \longrightarrow D M_{-}^{\mathrm{eff}}(E(G, \mathfrak{H}))
$$

(see $\S 3$ ). We now give our main result concerning strata in the Baily-Borel compactification.

Theorem 7.1. Assume that $(G, \mathfrak{H})$ satisfies $(+)$, and that $K$ is neat. Then there is a canonical isomorphism

$$
M_{\mathrm{gm}}\left(S_{1}^{K}, i_{g}^{!} j ! \mathbb{Z}\right) \stackrel{\sim}{\longrightarrow} R \Gamma\left(\Delta_{1}, \operatorname{Hom}\left(\mathbb{Z}\left(U_{1}(\mathbb{R})\right), M_{\mathrm{gm}}\left(S^{K_{1}}\right)\right)\right)\left[u_{1}\right]
$$

in $D M_{-}^{\mathrm{eff}}(E(G, \mathfrak{H}))$.

Proof. Choose a $K$-admissible cone decomposition $\mathfrak{S}$ satisfying the conditions of [Pin92, $\S(3.9)]$. In particular, all cones occurring in $\mathfrak{S}$ are smooth, and the decomposition is complete. Let us denote by $S^{K}(\mathfrak{S}):=S^{K}(G, \mathfrak{H}, \mathfrak{S})$ the toroidal compactification associated to $\mathfrak{S}$. It is a smooth projective scheme over $E(G, \mathfrak{H})$. The identity on $S^{K}$ extends uniquely to a surjective proper morphism

$$
p=p_{\mathfrak{S}}: S^{K}(\mathfrak{S}) \rightarrow\left(S^{K}\right)^{*} .
$$




\section{J. Wildeshaus}

Denote by $Z^{\prime}$ the inverse image under $p$ of the stratum $S_{1}^{K}$, by $i_{\mathfrak{S}}$ the immersion of $Z^{\prime}$ into $S^{K}(\mathfrak{S})$, and by $j_{\mathfrak{S}}$ the open immersion of $S^{K}$. We apply invariance under abstract blow-up [Wil06, Theorem 4.1], which tells us that

$$
p: M_{\mathrm{gm}}\left(Z^{\prime}, i_{\mathfrak{S}}^{!} j_{\mathfrak{S} !} \mathbb{Z}\right) \longrightarrow M_{\mathrm{gm}}\left(S_{1}^{K}, i_{g}^{!} j ! \mathbb{Z}\right)
$$

is an isomorphism. In order to identify the left-hand side, we now set up data (1)-(6) as in $\S 4$. The factorization of $\pi:\left(P_{1}, \mathfrak{X}_{1}\right) \rightarrow\left(G_{1}, \mathfrak{H}_{1}\right)$ corresponding to the weight filtration of the unipotent radical $W_{1}$ gives the following:

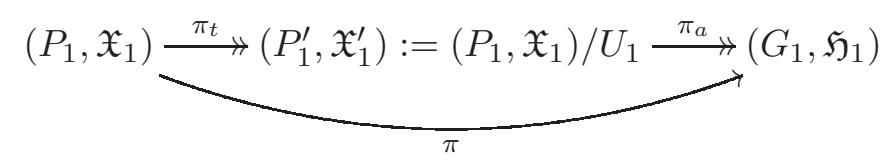

On the level of Shimura varieties, we obtain

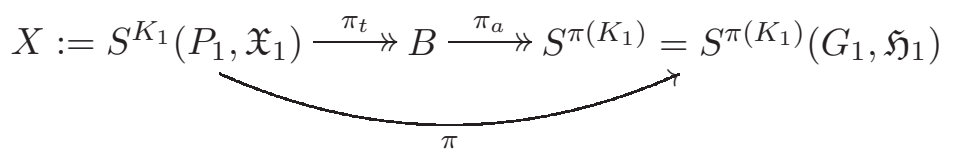

By [Pin90, SS3.12-3.22(a)], $\pi_{a}$ is in a natural way a torsor under an Abelian scheme, while $\pi_{t}$ is a torsor under a split torus $T$ of dimension $u_{1}$. Then [Pin92, $\left.\S(3.10)\right], \mathfrak{S}$ and the choice of the stratum $S_{1}^{K}$ induce a rational partial polyhedral decomposition of $Y_{*}(T)_{\mathbb{R}}$, equally denoted by $\mathfrak{S}$, and a non-empty proper subset $\mathfrak{T}$ of $\mathfrak{S}$ satisfying conditions $(\alpha)-(\gamma)$ from $\S 4$. In addition, the subset $D$ of $Y_{*}(T)_{\mathbb{R}}$ is contractible and open. As for the group, we take $H:=\Delta_{1}$. Its action on $B, X$, and $T$ is the action induced from the natural action of $H_{Q}$ on the Shimura data involved in the above factorization of $\pi$. It satisfies condition $(\delta)$. Finally, we set $S:=S^{K}, S_{\mathfrak{S}}:=S^{K}(\mathfrak{S})$, and $S_{\mathfrak{T}}:=Z^{\prime}$. The isomorphisms

$$
H \backslash\left(X_{\mathfrak{S}}\right)_{X_{\mathfrak{T}}} \stackrel{\sim}{\longrightarrow}\left(S_{\mathfrak{S}}\right)_{S_{\mathfrak{T}}}, \quad H \backslash\left(X_{\mathfrak{S}-\{0\}}\right)_{X_{\mathfrak{T}}} \stackrel{\sim}{\longrightarrow}\left(S_{\mathfrak{S}-\{0\}}\right)_{S_{\mathfrak{T}}}, \quad H \backslash X_{\mathfrak{T}} \stackrel{\sim}{\longrightarrow} S_{\mathfrak{T}}
$$

are those of [Pin92, p. 224]. Now apply Theorem 4.6(c).

By passing to simultaneous refinements of two cone decompositions, one sees that our isomorphism does not depend on the choice of $\mathfrak{S}$.

By [Wil06, Remark 3.2], the left-hand side of the isomorphism from Theorem 7.1 lies in $D M_{\mathrm{gm}}^{\mathrm{eff}}(E(G, \mathfrak{H}))$, and hence in the category $D M_{\mathrm{gm}}(E(G, \mathfrak{H}))$ of geometrical motives over $E(G, \mathfrak{H})$. Recall the functor

$$
L \Lambda\left(\Delta_{1}, \bullet\right): \Delta_{1}-D M_{-}^{\mathrm{eff}}(E(G, \mathfrak{H})) \longrightarrow D M_{-}^{\mathrm{eff}}(E(G, \mathfrak{H}))
$$

from $\S 3$.

Corollary 7.2. Assume that $(G, \mathfrak{H})$ satisfies $(+)$, and that $K$ is neat. Then there is a canonical isomorphism

$$
M_{\mathrm{gm}}^{c}\left(S_{1}^{K}, i_{g}^{*} j_{*} \mathbb{Z}\right) \stackrel{\sim}{\longrightarrow} L \Lambda\left(\Delta_{1}, \operatorname{Hom}\left(\mathbb{Z}\left(U_{1}(\mathbb{R})\right), M_{\mathrm{gm}}^{c}\left(S^{K_{1}}\right)\right)\right)\left[-u_{1}\right]
$$

in $D M_{\mathrm{gm}}^{\mathrm{eff}}(E(G, \mathfrak{H}))$.

Proof. By definition [Wil06, Definition 7.1], the left-hand side of the desired isomorphism is dual to

$$
M_{\mathrm{gm}}\left(S_{1}^{K}, i_{g}^{!} j ! \mathbb{Z}\right)\left(-\operatorname{dim} S^{K}\right)\left[-2 \operatorname{dim} S^{K}\right] .
$$

Let us compute the dual of the right-hand side, twisted by $\operatorname{dim} S^{K}$ and shifted by $2 \operatorname{dim} S^{K}-u_{1}$. In order to simplify notation, let us agree to drop the self-dual group $\mathbb{Z}\left(U_{1}(\mathbb{R})\right)$. Recall that the object $L \Lambda\left(\Delta_{1}, M_{\mathrm{gm}}^{c}\left(S^{K_{1}}\right)\right)$ of $D M_{-}^{\mathrm{eff}}(E(G, \mathfrak{H}))$ is represented by the complex $\left(F_{*} \otimes_{\mathbb{Z}} \underline{C}_{*}\left(L^{c}\left(S^{K_{1}}\right)\right)\right)_{\Delta_{1}}$, 


\section{ON THE BOUNDARY MOTIVE OF A SHIMURA VARIETY}

where $L^{c}\left(S^{K_{1}}\right)$ denotes the Nisnevich sheaf with transfers of quasi-finite correspondences, and where $F_{*} \rightarrow \mathbb{Z}$ is a bounded resolution of the trivial $\mathbb{Z} \Delta_{1}$-module $\mathbb{Z}$ by free $\mathbb{Z} \Delta_{1}$-modules. By adjunction,

$$
\underline{\operatorname{Hom}}\left(L \Lambda\left(\Delta_{1}, M_{\mathrm{gm}}^{c}\left(S^{K_{1}}\right)\right), \mathbb{Z}\left(\operatorname{dim} S^{K}\right)\left[2 \operatorname{dim} S^{K}\right]\right)
$$

is thus represented by $\operatorname{Hom}\left(F_{*}, z^{*}\left(\bullet, S^{K_{1}}\right)\right)^{\Delta_{1}}$, for any complex $z^{*}\left(\bullet, S^{K_{1}}\right)$ in

$$
C^{-}\left(\Delta_{1}-\operatorname{Shv}{ }_{N i s}(\operatorname{SmCor}(E(G, \mathfrak{H})))\right)
$$

representing

$$
\underline{\operatorname{Hom}}\left(M_{\mathrm{gm}}^{c}\left(S^{K_{1}}\right), \mathbb{Z}\left(\operatorname{dim} S^{K_{1}}\right)\left[2 \operatorname{dim} S^{K_{1}}\right]\right)
$$

(recall that $\left.\operatorname{dim} S^{K}=\operatorname{dim} S^{K_{1}}\right)$. We claim that the class of $z^{*}\left(\bullet, S^{K_{1}}\right)$ is canonically isomorphic to the $\Delta_{1}$-equivariant motive $M_{\mathrm{gm}}\left(S^{K_{1}}\right)$. Admitting this for the moment, we apply Corollary 3.3 to get a canonical isomorphism

$$
\underline{\operatorname{Hom}}\left(L \Lambda\left(\Delta_{1}, M_{\mathrm{gm}}^{c}\left(S^{K_{1}}\right)\right), \mathbb{Z}\left(\operatorname{dim} S^{K}\right)\left[2 \operatorname{dim} S^{K}\right]\right) \stackrel{\sim}{\rightarrow} R \Gamma\left(\Delta_{1}, M_{\mathrm{gm}}\left(S^{K_{1}}\right)\right) .
$$

However, by Theorem 7.1 , the right-hand side is isomorphic to $M_{\mathrm{gm}}\left(S_{1}^{K}, i_{g}^{!} j ! \mathbb{Z}\right)\left[-u_{1}\right]$. To conclude the proof, it remains to identify the object

$$
\underline{\operatorname{Hom}}\left(M_{\mathrm{gm}}^{c}\left(S^{K_{1}}\right), \mathbb{Z}\left(\operatorname{dim} S^{K_{1}}\right)\left[2 \operatorname{dim} S^{K_{1}}\right]\right)
$$

of $\Delta_{1}-D M_{-}^{\text {eff }}(E(G, \mathfrak{H}))$. One first constructs an isomorphism

$$
\underline{\operatorname{Hom}}\left(M_{\mathrm{gm}}\left(S^{K_{1}}\right), \mathbb{Z}\left(\operatorname{dim} S^{K_{1}}\right)\left[2 \operatorname{dim} S^{K_{1}}\right]\right) \stackrel{\sim}{\longrightarrow} M_{\mathrm{gm}}^{c}\left(S^{K_{1}}\right)
$$

as in the proof of [Voe00, Theorem 4.3.7,3.]. This requires a $\Delta_{1}$-equivariant version of duality for bivariant cycle cohomology, which is provided by [Ser01, Theorem 3.21(i) and its proof]. Next, adjunction gives a canonical morphism

$$
M_{\mathrm{gm}}\left(S^{K_{1}}\right) \longrightarrow \underline{\operatorname{Hom}}\left(M_{\mathrm{gm}}^{c}\left(S^{K_{1}}\right), \mathbb{Z}\left(\operatorname{dim} S^{K_{1}}\right)\left[2 \operatorname{dim} S^{K_{1}}\right]\right)
$$

in $\Delta_{1}-D M_{-}^{\text {eff }}(E(G, \mathfrak{H}))$. That it is an isomorphism can be checked in the category $D M_{-}^{\text {eff }}(E(G, \mathfrak{H}))$ (see [Ser01, Proposition 3.1]). Now apply [Voe00, Theorem 4.3.7, 3.].

A more conceptual proof of Corollary 7.2 could be given if the category $\Delta_{1}-D M_{\operatorname{gm}}(E(G, \mathfrak{H}))$ were known to be a rigid tensor category (see Remark 3.10), in which the $\Delta_{1}$-equivariant motive is dual to the $\Delta_{1}$-equivariant motive with compact support (with the usual twist and shift).

Theorem 7.1 and Corollary 7.2 identify the motives occurring as graded pieces in the colocalization [Wil06, §3] (respectively, localization [Wil06, §7]) filtration of the boundary motive $\partial M_{\mathrm{gm}}\left(S^{K}\right)$. Here, the filtration is associated to the stratification of the Baily-Borel compactification.

The isomorphisms from Theorem 7.1 and Corollary 7.2 induce isomorphisms for motivic cohomology

$$
H^{i}(X, \mathbb{Z}(j)):=\operatorname{Hom}_{D M_{\mathrm{gm}}}\left(M_{\mathrm{gm}}(X), \mathbb{Z}(j)[i]\right)
$$

and for motivic cohomology with compact support

$$
H_{c}^{i}(X, \mathbb{Z}(j)):=\operatorname{Hom}_{D M_{\mathrm{gm}}}\left(M_{\mathrm{gm}}^{c}(X), \mathbb{Z}(j)[i]\right),
$$

for $i, j \in \mathbb{Z}$, or more generally, isomorphisms in any category equipped with a functor from $D M_{\mathrm{gm}}^{\mathrm{eff}}(E(G, \mathfrak{H}))$. Let us spell out, for example, what Corollary 7.2 implies for motivic cohomology.

COROLlary 7.3. In the situation of Corollary 7.2, there is a canonical isomorphism of Abelian groups between

$$
\operatorname{Hom}_{D M_{\mathrm{gm}}(E(G, \mathfrak{H}))}\left(M_{\mathrm{gm}}^{c}\left(S_{1}^{K}, i_{g}^{*} j_{*} \mathbb{Z}\right), \mathbb{Z}(j)[i]\right)
$$




\section{J. Wildeshaus}

and

$$
H_{c}^{i+u_{1}}\left(S^{K_{1}}, \Delta_{1}, \mathbb{Z}(j)\right) \otimes_{\mathbb{Z}} \mathbb{Z}\left(U_{1}(\mathbb{R})\right),
$$

for $i, j \in \mathbb{Z}$. Here, the $H_{c}^{*}\left(\bullet, \Delta_{1}, \mathbb{Z}(j)\right)$ denote equivariant motivic cohomology with compact support, i.e. the cohomology associated to the composition

$$
R \Gamma\left(\Delta_{1}, \bullet\right) \circ R \operatorname{Hom}_{D M_{-}^{\mathrm{eff}}(E(G, \mathfrak{H}))}(\bullet, \mathbb{Z}(j)) \circ M_{\mathrm{gm}}^{c}
$$

mapping $\Delta_{1}-S c h / E(G, \mathfrak{H})$ to the derived category of Abelian groups.

Remark 7.4. The analogous formula for étale cohomology holds thanks to the existence of the étale realization functor $[$ Voe00, $§ 3.3]$. Note that equivariant étale cohomology

$$
H_{c, \text { et }}^{i+u_{1}}\left(S^{K_{1}}, \Delta_{1}, \mathbb{Z} / n \mathbb{Z}(j)\right) \otimes_{\mathbb{Z}} \mathbb{Z}\left(U_{1}(\mathbb{R})\right)
$$

equals

$$
H_{c, \mathrm{et}}^{i+u_{1}}\left(S^{\pi\left(K_{1}\right)}, \Delta_{1}, R \pi ! \mathbb{Z} / n \mathbb{Z}(j)\right) \otimes_{\mathbb{Z}} \mathbb{Z}\left(U_{1}(\mathbb{R})\right) .
$$

Using duality for the cohomology of unipotent groups, and for $\pi_{*}$ and $\pi_{\text {! }}$, we get canonically

$$
H_{c, \text { et }}^{i+u_{1}}\left(S^{K_{1}}, \Delta_{1}, \mathbb{Z} / n \mathbb{Z}(j)\right) \otimes_{\mathbb{Z}} \mathbb{Z}\left(U_{1}(\mathbb{R})\right) \cong H_{c, \text { et }}^{i}\left(S^{\pi\left(K_{1}\right)}, \Delta_{1}, R \pi_{*} \mathbb{Z} / n \mathbb{Z}(j)\right) .
$$

The resulting isomorphism

$$
H_{c, \text { et }}^{i}\left(S_{1}^{K}, i_{g}^{*} j_{*} \mathbb{Z} / n \mathbb{Z}(j)\right) \stackrel{\sim}{\longrightarrow} H_{c, \text { et }}^{i}\left(S^{\pi\left(K_{1}\right)}, \Delta_{1}, R \pi_{*} \mathbb{Z} / n \mathbb{Z}(j)\right)
$$

should be identical to that obtained by applying the functor $H_{c \text {,et }}^{i}$ to the comparison isomorphism from the main result of [Pin92] in the special case of Tate coefficients $\mathbb{Z} / n \mathbb{Z}(j)$. In the same way, but by applying the étale realization to the isomorphism in $D M_{\mathrm{gm}}^{\mathrm{eff}}(\bar{E})$ induced by that from Corollary 7.2 $(\bar{E}:=$ the algebraic closure of $E(G, \mathfrak{H}))$, one obtains an isomorphism

$$
H_{c, \text { et }}^{i}\left(S_{1, \bar{E}}^{K}, i_{g}^{*} j_{*} \mathbb{Z} / n \mathbb{Z}(j)\right) \stackrel{\sim}{\longrightarrow} H_{c, \text { et }}^{i}\left(S_{\bar{E}}^{\pi\left(K_{1}\right)}, \Delta_{1}, R \pi_{*} \mathbb{Z} / n \mathbb{Z}(j)\right)
$$

of modules under the absolute Galois group of $E(G, \mathfrak{H})$. Again, this isomorphism should be compared with that deduced from the main result of [Pin92].

Remark 7.5. There is a motivic version of Theorem 7.1 for more general than constant coefficients $\mathbb{Z}$. More precisely, one fixes mixed Shimura data $(P, \mathfrak{X})$ whose underlying pure data are $(G, \mathfrak{H})$. Choose an open compact subgroup $K^{P} \subset P\left(\mathbb{A}_{f}\right)$ mapping onto $K$ under the projection $p$ from $(P, \mathfrak{X})$ to $(G, \mathfrak{H})$. One defines the motive $M_{\mathrm{gm}}\left(S_{1}^{K}, i_{g}^{!} j ! p ! \mathbb{Z}\right)$ by using a toroidal compactification of the mixed Shimura variety $S^{K^{P}}(P, \mathfrak{X})$ (one shows that the choice of compactification does not matter). The general version of the isomorphism of Theorem 7.1 then takes the following form:

$$
M_{\mathrm{gm}}\left(S_{1}^{K}, i_{g}^{!} j ! p ! \mathbb{Z}\right) \stackrel{\sim}{\longrightarrow} R \Gamma\left(\Delta_{1}^{P}, \operatorname{Hom}\left(\mathbb{Z}\left(U_{1}^{P}(\mathbb{R})\right), M_{\mathrm{gm}}\left(S^{K_{1}^{P}}\right)\right)\right)\left[u_{1}^{P}\right] .
$$

Here, $U_{1}^{P}$ is the 'weight -2 ' part of the boundary component $\left(P_{1}^{P}, \mathfrak{X}_{1}^{P}\right)$ of $(P, \mathfrak{X})$ lying over $\left(P_{1}, \mathfrak{X}_{1}\right)$, $u_{1}^{P}$ is its dimension, and $S^{K_{1}^{P}}$ is the Shimura variety associated to $\left(P_{1}^{P}, \mathfrak{X}_{1}^{P}\right)$. Finally, $\Delta_{1}^{P}$ is defined in exact analogy to $\Delta_{1}$, by replacing $Q, P_{1}, K$, and $\mathfrak{H}_{1}$ by the respective groups and spaces occurring in the description of the boundary component $\left(P_{1}^{P}, \mathfrak{X}_{1}^{P}\right)$. Details will be published elsewhere.

\section{ACKNowledgements}

This work was done while I was enjoying a délégation auprès $d u C N R S$, and during visits to the Sonderforschungsbereich 478 of the University of Münster, and to the Moulin des traits at CinqMars-la-Pile. I am grateful to all three institutions. I also wish to thank D. Blottière and F. Lemma for useful discussions and comments. 


\section{ON THE BOUNDARY MOTIVE OF A SHIMURA VARIETY}

\section{REFERENCES}

AMRT75 A. Ash, D. Mumford, M. Rapoport and Y. Tai, Smooth compactification of locally symmetric varieties, in Lie groups: history, frontiers and applications, vol. IV (MathSci Press, Brookline, MA, 1975).

BB66 W. L. Baily and A. Borel, Compactification of arithmetic quotients of bounded symmetric domains, Ann. of Math. (2) 84 (1966), 442-528.

BS73 A. Borel and J.-P. Serre, Corners and arithmetic groups, Comment. Math. Helv. 48 (1973), 436-491.

BW04 J. I. Burgos and J. Wildeshaus, Hodge modules on Shimura varieties and their higher direct images in the Baily-Borel compactification, Ann. Sci. École Norm. Sup. (4) 37 (2004), 363-413.

Har86 G. Harder, Eisenstein-Kohomologie arithmetischer Gruppen: Allgemeine Aspekte, Preprint, University of Bonn (1986).

HZ94 M. Harris and S. Zucker, Boundary cohomology of Shimura varieties II. Hodge theory at the boundary, Invent. Math. 116 (1994), 243-307.

KKMS73 G. Kempf, F. Knudsen, D. Mumford and B. Saint-Donat, Toroidal embeddings. I, Lecture Notes in Mathematics, vol. 339 (Springer, Berlin, 1973).

LR91 E. Looijenga and M. Rapoport, Weights in the local cohomology of a Baily-Borel compactification, in Complex geometry and Lie theory, Proceedings of Symposia in Pure Mathematics, vol. 53, eds J. A. Carlson, C. H. Clemens and D. R. Morrison (American Mathematical Society, Providence, RI, 1991), 223-260.

Moo98 B. Moonen, Models of Shimura varieties in mixed characteristics, in Galois representations in arithmetic algebraic geometry, London Mathematical Society Lecture Notes, vol. 254 (Cambridge University Press, Cambridge, 1998), 267-350.

Pin90 R. Pink, Arithmetical compactification of mixed Shimura varieties, Bonner Mathematische Schriften, vol. 209 (University of Bonn, Bonn, 1990).

Pin92 R. Pink, On $\ell$-adic sheaves on Shimura varieties and their higher direct images in the Baily-Borel compactification, Math. Ann. 292 (1992), 197-240.

Ser01 C. Serpé, A triangulated category of equivariant motives, Preprint, SFB 478 Preprint Series, Heft 173, University of Münster (2001); http://wwwmath.uni-muenster.de/u/serpe/arbeiten.html.

Ver77 J.-L. Verdier, Catégories dérivées, état 0, in Séminaire de Géométrie Algébrique (SGA $4 \frac{1}{2}$ ), Lecture Notes in Mathematics, vol. 569 (Springer, Berlin, 1977), 262-311.

Voe00 V. Voevodsky, Triangulated categories of motives, in Cycles, transfers, and motivic homology theories, Annals of Mathematical Studies, vol. 143, eds V. Voevodsky, A. Suslin and E. M. Friedlander (Princeton University Press, Princeton, NJ, 2000).

Wil00 J. Wildeshaus, Mixed sheaves on Shimura varieties and their higher direct images in toroidal compactifications, J. Algebraic Geom. 9 (2000), 323-353.

Wil06 J. Wildeshaus, The boundary motive: definition and basic properties, Compositio Math. 142 (2006), 631-656.

Jörg Wildeshaus wildesh@math.univ-paris13.fr

LAGA, Institut Galilée, Université Paris 13, Avenue Jean-Baptiste Clément, F-93430 Villetaneuse, France 